\title{
Lattice Gauge Fields, Principal Bundles and the Calculation of Topological Charge
}

\author{
Anthony Phillips ${ }^{1 \star}$ and David Stone \\ 1 Department of Mathematics, State University of New York at Stony Brook, Stony Brook, \\ NY 11794, USA \\ 2 Department of Mathematics, Brooklyn College, Brooklyn, NY11210, USA
}

\begin{abstract}
SU}_{2}$-valued lattice gauge fields are studied on a 4-dimensional simplicial lattice. If $\mathbf{u}$ has sufficiently small plaquette products, then there is a unique principal $\mathrm{SU}_{2}$-bundle $\xi$ admitting transition functions, defined on the intersections of adjacent dual cells, which take values within $\pi / 8$ of $\mathbf{u}$. An algorithm is explicitly given which associates an integer to every $\mathbf{u}$ off a certain set of measure zero. This algorithm only involves evaluation of $4 \times 4$ determinants and the solution of quadratic equations. When $\mathbf{u}$ is as above, the integer produced is the second Chern number of $\xi$, i.e. the topological charge of $\mathbf{u}$.
\end{abstract}

\section{Introduction}

This article has a theoretical and a practical side. It analyzes the circumstances under which an $\mathrm{SU}_{2}$-valued lattice gauge field determines a principal $\mathrm{SU}_{2}$-bundle, and it also presents an explicit algorithm which then computes the second Chern number of that bundle, the topological charge, directly from the lattice data. This algorithm, which involves no more than $4 \times 4$ determinants and the solution of quadratic equations, has been used (in joint work with Gordon Lasher) for a Monte Carlo calculation of topological susceptibility; the details and results are reported elsewhere [15].

For early work on this problem, see [5, 6], and also $[13,17]$; our work grew out of an attempt to find an appropriate mathematical context for Martin Lüscher's construction [18]. Lüscher's algorithm has recently been programmed [9]; other topological charge algorithms have been given in [14, 24, 30,31], and have been discussed in [21,22].

Lattice gauge fields were introduced by Kenneth Wilson in 1974 [29] (see also [25]) to represent classical field configurations in Monte Carlo evaluations of path integral solutions of quantum field theories. Here is the context. (We shall assume for simplicity in this work that we are dealing with a compact space-time $X$; as usual, the time coordinate has been rotated in the complex plane to give $X$ a Euclidean metric.) Let us fix a compact Lie group $G$. The set of gauge fields on $X$ 
with group $G$, which is the set over which the Feynman-type integration would have to be carried out, has the following properties. It has (in general) infinitely many connected components, one for each principal $G$-bundle over $X$. Each connected component is the space of gauge fields, or connections, in the corresponding bundle. As a function space it is infinite-dimensional. Wilson replaces $X$ with a finite cell complex, or lattice, $\Lambda$ (cubical in his formulation), admitting as variables only the symbols associated to the various-dimensional cells of $\Lambda$; in all, a finite collection. A gauge field on $X$ is represented, once fiber coordinates have been chosen at the vertices $\alpha, \beta, \ldots$ of $\Lambda$, by the parallel transport it induces along each 1 -cell $\langle\alpha \beta\rangle$ of $\Lambda$. This corresponds to an element $u_{\alpha \beta}$ of $G$ (these "transporters" clearly satisfy $u_{\beta \alpha}=u_{\alpha \beta}^{-1}$ ) and any such assignment $\mathbf{u}$ of group elements to 1 -cells is called a $G$-valued lattice gauge field on $\Lambda$. Thus the infinite collection of infinite dimensional function spaces has been replaced by the compact, finite-dimensional set $G \times \ldots \times G$, one factor for each 1-cell in $\Lambda$.

Mathematically speaking, this procedure is somewhat mysterious, and it seemed all the more so when for $G=\mathrm{SU}_{N}$ one of the quantities calculated by integrating over $G \times \ldots \times G$ (i.e., computing the path integral) was the topological susceptibility $\left\langle Q^{2}\right\rangle$ /Volume; $Q$ is the topological charge, which is known to be a bundle invariant, in fact equal to the second Chern number. Does this mean that a non-trivial bundle can be defined without reference to coordinate systems? The answer is almost yes.

First of all it is fairly obvious that since $Q$ is an integer-valued function on the connected space $G \times \ldots \times G$, any reasonable algorithm assigning bundles to lattice gauge fields must be discontinuous on some subset $K$ of $G \times \ldots \times G$, and locally constant elsewhere. It turns out that once an appropriate set $K$ has been excluded, then every remaining lattice gauge field can be interpreted as determining a principal $G$-bundle over $\Lambda$, and therefore as having a well-defined topological charge. This is proved in [23] for $G=U_{1}$ (see also [10]); there the definition of $K$ is very simple: a lattice gauge field belongs to $K$ if any one of its plaquette products equals -1 .

The corresponding statement for $G=\mathrm{SU}_{2}$ is the main theoretical result of this work. In this case it is most convenient to work with a simplicial lattice $[2-4,7]$ (this greatly simplifies the dual geometry); the plaquettes are now triangular. The set $K$ still has measure zero, but its definition is less simple and perhaps less natural, because the algorithm we use to construct a bundle from a lattice gauge field requires additional information: the choice of a local ordering $\mathbf{o}$ of the vertices of $\Lambda$. The excluded set $K$ depends on $\mathbf{0}$, and a single lattice gauge field may determine different bundles with different orderings; see Example 3.20. (It may be that such a choice is unavoidable in working with a non-abelian gauge group; for evidence, besides the other topological charge algorithms referred to above, see [16]. This pathology does not occur with lattice gauge fields which are sufficiently smooth: we will prove that then there is an algorithm-independent closest principal $\mathrm{SU}_{2}$-bundle. Here is the exact statement. (Complete definitions are given in Sect. 2.)

Theorem A. Let $\mathbf{u}$ be an $\mathrm{SU}_{2}$-valued lattice gauge field defined on a 4-dimensional simplicial complex $\Lambda$. Suppose $\mathbf{u}$ satisfies hypothesis $H 1$ or the stronger $\mathrm{H} 2$. 
H1. The product of the transporters along any simple closed edge path in any simplex of $\Lambda$ is within $\pi / 8$ of the identity.

H2. Each plaquette product of $\mathbf{u}$ is within $\pi / 24$ of the identity (distances are in the unit-sphere metric on $\mathrm{SU}_{2}$ ).

Then there exists a unique principal $\mathrm{SU}_{2}$-bundle $\xi$ with the following property: $\xi$ can be trivialized over the 4-dimensional dual cells $\left\{c_{\mu}\right\}$ of $\Lambda$ in such a way that, for each pair $\alpha, \beta$ of adjacent vertices, the transition function $v_{\alpha \beta}: c_{\alpha} \cap c_{\beta} \rightarrow \mathrm{SU}_{2}$ relating the fiber coordinates over $c_{\alpha}$ and $c_{\beta}$ takes values in the ball of radius $\pi / 8$ about the transporter $u_{\alpha \beta}$.

The proof of this theorem occupies Sect. 2 (existence) and Sect. 3 (uniqueness) below. The existence proof uses an explicit algorithm to construct a set of transition functions. In addition we will prove (Theorem 3.6) that, under the hypothesis derived from $\mathrm{H} 1$ by replacing $\pi / 8$ with $\pi / 2$, the bundle produced by this algorithm does not depend on the local ordering. The $\pi / 2$ bound can be weakened slightly; see (3.19); but Example 3.20 shows a lattice gauge field satisfying $\mathrm{H} 1$ with $\pi / 8$ replaced by approximately $2 \pi / 3$, to which our algorithm, with two different orderings, assigns two different bundles.

Our more practical results solve the problem of identifying the bundle constructed from an $\mathrm{SU}_{2}$-valued lattice gauge field by our algorithm, i.e. of calculating its topological charge. This work is in Sect. 4. We will show that an extension of our bundle-algorithm leads to a rule assigning an integer to every lattice gauge field $\mathbf{u}$ in the complement of a larger set $K^{\prime}$ (still of measure zero). This integer is the second Chern number of the corresponding principal bundle, i.e. the topological charge of $\mathbf{u}$.

From the mathematical point of view, this study of lattice gauge fields yields a new way of computing the second Chern number of a principal $\mathrm{SU}_{2}$-bundle $\xi$ over a triangulated 4-manifold $M$, if $\xi$ has a connection $\omega$; this method works almost always when the curvature of $\omega$ is sufficiently small relative to the triangulation. Namely, if $M$ is triangulated as a simplicial complex $\Lambda$, there is a straightforward way of using the linear structure of the simplexes of $\Lambda$ and parallel transport by $\omega$ to trivialize $\xi$ over each dual cell $c_{\mu}$, once a fiber coordinate has been chosen at the vertex $\mu$. The set $\mathbf{v}$ of transition functions relating these trivializations is given by $v_{\alpha \beta}(x)=$ the group element (in the vertex $\beta$ coordinate) reached by paralleltransporting $I$ (at vertex $\alpha$ ) by $\omega$ along the broken path $\alpha x \beta$. Let $p_{\alpha \beta}=\langle\alpha \beta\rangle \cap c_{\alpha}$ $\cap c_{\beta}$. If we define a lattice gauge field $\mathbf{u}$ on $\Lambda$ by $u_{\alpha \beta}=v_{\alpha \beta}\left(p_{\alpha \beta}\right)$ (this is in fact the standard way of constructing a lattice gauge field from $\omega$ ) then $\mathbf{u}$ and $\mathbf{v}$ will be related as in the conclusion of Theorem A as soon as $(*)$ parallel transport by $\omega$ around the triangular path $\alpha x \beta \alpha$ takes $I$ back to within $\pi / 8$ of itself, for every $x$ in $c_{\alpha} \cap c_{\beta}$. Then the uniqueness part of Theorem A guarantees that the integer calculated by applying the algorithm of Sect. 4 to $\mathbf{u}$ is in fact $C_{2}(\xi)$. This should be compared with the usual calculation $[8,19]$ of $C_{2}(\xi)$ from $\omega$, namely

$$
C_{2}(\xi)=\left(1 / 8 \pi^{2}\right) \int_{M} \operatorname{tr}(\Omega \wedge \Omega)=\left(-1 / 4 \pi^{2}\right) \int_{M} \operatorname{det} \Omega,
$$

where $\Omega$ is the curvature 2-form of $\omega$. The algorithm rejects a measure-zero set of u's, hence the "almost always" above.

It is fairly clear that (*) can be guaranteed by controlling $\Omega$. More precisely, let $T$ represent the triangle with edge-path $\partial T=\alpha x \beta \alpha$. Then the element reached by 
parallel-transporting $I$ around $\partial T$ may be written as $P \int_{\partial T} \omega$, where $P \int$ represents the path-ordered or "product" integral. There is a product-integral version of Stokes' Theorem $[20,26]$, which we may write symbolically as $(* *) P \int_{\partial T} \omega=P \iint_{T} \Omega$.

The point is that if we choose a Riemannian metric on $M$, and let $\|\Omega\|(p)$ $=\sup |\Omega(v, w)|$, where for $A \in \mathfrak{s u}_{2}$, written as $A=a_{1} \mathbf{i}+a_{2} \mathbf{j}+a_{3} \mathbf{k},|A|=\left(\sum a_{i}^{2}\right)^{1 / 2}$ and the supremum is taken over all orthonormal pairs $v, w$ of tangent vectors at $p$, then it is straightforward to deduce from $(* *)$ that the distance from $I$ to $P \int_{\partial T} \omega$ is bounded by $\iint_{T}\|\Omega\| d A$.

\section{Remarks on the Method of Proof}

It will become clear to the reader that our theoretical and practical results are all attained by the same general proof-scheme. The scheme is quite simple, although the details of its implementation become somewhat elaborate. It has three parts.

Part one is the interpretation of a lattice gauge field $\mathbf{u}$ as giving, for each pair $c_{\alpha}$, $c_{\beta}$ of adjacent dual 4-cells, the value of a transition function $v_{\alpha \beta}: c_{\alpha} \cap c_{\beta} \rightarrow \mathrm{SU}_{2}$ at the point $p_{\alpha \beta}$, where the bond $\langle\alpha \beta\rangle$ intersects $c_{\alpha} \cap c_{\beta}$ :

$$
v_{\alpha \beta}\left(p_{\alpha \beta}\right)=u_{\alpha \beta}
$$

(see Fig. 2.1). The problem is then to extend this one value to a function defined on all of $c_{\alpha} \cap c_{\beta}$, in such a way as to satisfy the cocycle condition on triple intersections, while staying as close to $u_{\alpha \beta}$ as possible.

Second, one attacks this problem separately inside each 4-simplex $\sigma$ containing $\alpha$ and $\beta$. Inside $c_{\alpha} \cap c_{\beta} \cap \sigma$ the form of the expression giving $v_{\alpha \beta}$ depends on the relative position of $\alpha$ and $\beta$ in the ordering induced by $\boldsymbol{o}$ on the vertices of $\sigma$; the way it depends is dictated by the "as constant as possible" principle: if $\alpha$ and $\beta$ are orderadjacent in $\sigma$, then $v_{\alpha \beta} \equiv u_{\alpha \beta}$ on $c_{\alpha} \cap c_{\beta} \cap \sigma$; if there is exactly one vertex $\gamma$ orderintermediate between them, then $v_{\alpha \beta}$ only varies, on $c_{\alpha} \cap c_{\beta} \cap \sigma$, as a function of the (modified) barycentric coordinate corresponding to $\gamma$; etc.

Third, the way $v_{\alpha \beta}$ varies with whatever barycentric coordinates are required by the second part is determined geometrically and consists in mapping straight lines in $\sigma$ to unique shortest geodesics in $\mathrm{SU}_{2}$, proportionally to length. A configuration will belong to the set $K$ precisely when the unique shortest geodesics required here do not exist.

This scheme, which will be explained in detail in Sect. 2, is applied to the complex $\Lambda$ in order to define the bundle determined by a lattice gauge field; to the augmented complex $\hat{\Lambda}$ (the cone on $\Lambda$ ), defined by adding a new vertex and connecting it to all the simplexes of the lattice, in order to calculate its topological charge (the resulting algorithm is computationally efficient because Parts 2 and 3 of the proof-scheme above are geometrically so simple); and to the product complex $\Lambda \times[0,1]$ whenever it is necessary to prove that two bundles are the same.

\section{Statement and Proof of the Existence Theorem}

2.1. The main problem underlying the work in this section is the reconstruction (when possible) of an $\mathrm{SU}_{2}$-gauge field from an $\mathrm{SU}_{2}$-valued lattice gauge field 


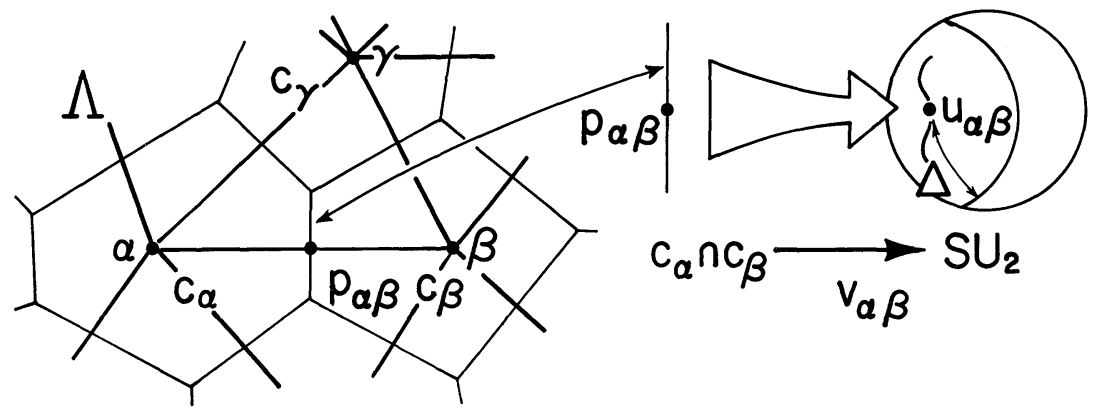

Fig. 2.1. Relationship between an $\mathrm{SU}_{2}$-valued lattice gauge field $\mathbf{u}$ and a coordinate $\mathrm{SU}_{2}$-bundle $\mathbf{v}$

defined on a 4-dimensional simplicial complex $\Lambda$. (At the end of Sect. 3 we shall indicate what modifications are necessary to extend this work to other gauge groups and higher-dimensional complexes.)

What we actually construct is an intermediate object.

2.2. Definition. Given a simplicial complex $A$ and a Lie group $G$, a coordinate G-bundle $\mathbf{v}$ on $\Lambda$ [27] is the assignment, to each pair $\alpha, \beta$ of adjacent vertices, of a continuous map $v_{\alpha \beta}: c_{\alpha} \cap c_{\beta} \rightarrow G$ defined on the intersection of the corresponding dual cells. These maps must satisfy the cocycle condition:

$$
\begin{gathered}
v_{\beta \alpha}(x)=v_{\alpha \beta}(x)^{-1} \quad \text { for } \quad x \in c_{\alpha} \cap c_{\beta}, \\
v_{\alpha \gamma}(x)=v_{\alpha \beta}(x) v_{\beta \gamma}(x) \text { for } x \in c_{\alpha} \cap c_{\beta} \cap c_{\gamma} .
\end{gathered}
$$

A coordinate bundle $\mathbf{v}$ is in fact a mixed topological-geometric object: it has an underlying principal bundle $\xi$, and it determines a connection in $\xi$ up to the choice of a partition of unity $[1,23]$.

2.3. Our construction of $\mathbf{v}$ from a lattice gauge field $\mathbf{u}$ requires some additional information: a local ordering $\mathbf{0}$ of the vertices of $\Lambda$, i.e. a partial ordering of the vertices in which the vertices of every simplex are totally ordered.

2.4. Notation. If $\alpha, \beta, \gamma, \ldots, \delta, \varepsilon$ are vertices of a simplex of $\Lambda$, the notation $u_{\alpha \beta \gamma} \ldots \delta \varepsilon$ will represent the product $u_{\alpha \beta} u_{\beta \gamma} \ldots u_{\delta \varepsilon}$. Furthermore if $\sigma$ is a simplex of $\Lambda, u_{\mathbf{o}}(\sigma)$ will represent the product $u_{\alpha \beta \ldots \zeta \alpha}$, where $\alpha<\beta<\ldots<\zeta$ are the vertices of $\sigma$ as ordered by $\mathbf{o}$.

We identify $\mathrm{SU}_{2}$ with the group of unit quaternions; geometrically this is the unit sphere in $\mathbf{R}^{4}$. We denote the distance between $X$ and $Y$ in the unit-sphere metric by $d(X, Y)$. The identity element of $\mathrm{SU}_{2}$ is represented by $I$ or $\mathbf{1}$.

If $\alpha$ and $\beta$ are adjacent vertices, let $p_{\alpha \beta}=\langle\alpha \beta\rangle \cap c_{\alpha} \cap c_{\beta}$ be the point where the bond between them intersects the common face of their dual cells. (Note that we are using the topological dual, described explicitly in (2.9) below, and not the metric dual of [2], so each simplex intersects its dual in its barycenter. For the random lattices of [2], the two duals are isomorphic complexes, but their relative positions with respect to a given lattice may be different.)

2.5. Theorem. Let $\mathbf{u}$ be an $\mathrm{SU}_{2}$-valued lattice gauge field on a simplicial complex $\Lambda$, and let $\Delta$ be a positive number, $\Delta \leqq \pi / 2$. Suppose that there exists a local ordering $\mathbf{0}$ of 
the vertices of $\Lambda$ such that, for every simplex $\tau$ of $\Lambda, d\left(I, u_{\mathbf{o}}(\tau)\right)<\Delta$. Then there exists a coordinate $\mathrm{SU}_{2}$-bundle $\mathbf{v}$ on $\Lambda$ such that

$$
\begin{gathered}
v_{\alpha \beta}\left(p_{\alpha \beta}\right)=u_{\alpha \beta} \quad \text { for every adjacent pair } \alpha, \beta, \\
d\left(v_{\alpha \beta}(x), u_{\alpha \beta}\right)<\Delta \quad \text { for every } x \in c_{\alpha} \cap c_{\beta} .
\end{gathered}
$$

2.6. Remarks. 1. The existence part of Theorem A follows from Theorem 2.5 (with $\Delta=\pi / 8$ ). Since balls of radius $\Delta<\pi / 2$ in $\mathrm{SU}_{2}$ are geodesically convex, Theorem 2.5 will be an immediate consequence of Theorem 2.8 below.

2. The problem of constructing an $\mathrm{SU}_{2}$-coordinate bundle from $\mathbf{u}$ would become trivial, and its solution meaningless, if we required only that $\mathbf{v}$ satisfy (2.5.1); because in fact, given any principal $\mathrm{SU}_{2}$-bundle $\xi$ on $\Lambda$, such a coordinate bundle $\mathbf{v}$ can easily be constructed for $\xi$.

3. The variation $\Delta$ of $v_{\alpha \beta}$ on $c_{\alpha} \cap c_{\beta}$ cannot be made arbitrarily small while preserving (2.5.2) and (2.2.1). Suppose for example that there were a plaquette $\langle\alpha \beta \gamma\rangle$ in $\Lambda$ such that $d\left(I, u_{\alpha \beta \gamma \alpha}\right) \geqq 3 \Delta$. Then (2.5.2) and (2.2.1) would imply that, for

$$
x \in c_{\alpha} \cap c_{\beta} \cap c_{\gamma}, \quad d\left(v_{\alpha \beta}(x) v_{\beta \gamma}(x) v_{\gamma \alpha}(x), u_{\alpha \beta \gamma \alpha}\right)=d\left(I, u_{\alpha \beta \gamma \alpha}\right)<3 \Delta,
$$

giving a contradiction.

4. The $\mathbf{v}$ promised by this theorem is produced by an algorithm with a larger domain of application (see below). Moreover the exact nature of the functions $v_{\alpha \beta}$ generated by this algorithm is important, since it will allow a simple calculation of the topological charge of the underlying $\xi$.

2.7. Definition. Given a 4-dimensional simplicial complex $\Delta$, and a local ordering o of its vertices, let $K(\mathbf{o})$ be the set of $\mathrm{SU}_{2}$-valued lattice gauge fields on $\Lambda$ which fail to satisfy the following condition:

Continuity condition with respect to o: On every 4-simplex $\sigma=\langle 01234\rangle$ (the vertices are $\mathbf{0}$-ordered by their numbers), one or the other of these conditions is met by $\mathbf{u}$ :(If $\Lambda$ does not have the property that every simplex is a face of a 4-simplex, additional analogous conditions should be added for the lower-dimensional simplexes.)

Condition $A(\mathbf{o})$. Each of the following five sets of elements of $\mathrm{SU}_{2}$ is linearly independent in $\mathbf{R}^{4}$ :

$$
\begin{array}{llll}
I, & u_{0120}, & u_{01240}, & u_{012340}, \\
I, & u_{0140}, & u_{01240}, & u_{012340}, \\
I, & u_{0140}, & u_{01340}, & u_{012340}, \\
I, & u_{0340}, & u_{01340}, & u_{012340}, \\
I, & u_{0340}, & u_{02340}, & u_{012340}
\end{array}
$$

Condition $B(\mathbf{o})$. For each 2,3 or 4 -face $\tau$ of $\sigma$,

$$
d\left(I, u_{\mathbf{o}}(\tau)\right)<\pi / 2 \text {. }
$$

(I.e. the sixteen elements $u_{0120}, \ldots, u_{1231}, \ldots, u_{012340}$ are all within $\pi / 2$ of the identity.)

Note that $K(\mathbf{o})$ is gauge-invariantly defined; furthermore, it is a set of measure zero in the space of all lattice gauge fields on $\Lambda$, if that space is metrised as $\mathrm{SU}_{2} \times \ldots \times \mathrm{SU}_{2}$, one factor for each bond. 
2.8. Theorem. Suppose $\mathbf{u}$ does not belong to $K(\mathbf{0})$. Then there exists a $\mathbf{v}$ satisfying

$$
v_{\alpha \beta}\left(p_{\alpha \beta}\right)=u_{\alpha \beta}
$$

and the following condition.

If $x \in c_{\alpha} \cap c_{\beta} \cap \sigma$, let $n$ be the number of vertices of $\sigma$ which are o-between $\alpha$ and $\beta$. Then $v_{\alpha \beta}(x) u_{\alpha \beta}^{-1}$ is in the geodesic convex hull of the $2^{n}$ points $u_{\mathbf{0}}(\tau)$, where $\tau=\langle\alpha \ldots \beta\rangle$ is a face of $\sigma$ with lowest-ordered vertex $\alpha$ and highest-ordered vertex $\beta$.

[The $B(\mathbf{o})$ part of the hypothesis can be weakened slightly; see (3.19).] Theorem 2.8 will be proved after we have set down some additional definitions and conventions about notation.

2.9. Notation Regarding $\Lambda$; Modified Barycentric Coorindates; Geodesics. In this section we use $\alpha, \beta, \gamma, \delta, \varepsilon$, and $\lambda, \mu, v$ to denote vertices of $\Lambda$, and $\varrho, \sigma, \tau$ for simplices of dimension $\geqq 1$. For the sake of simplicity we suppose that $\operatorname{dim} \sigma=4$ in the rest of (2.9), but the terminology will also be used (with appropriate modifications) in case $\operatorname{dim} \sigma<4$ (and in later sections, when $\operatorname{dim} \sigma=5$ ). We write $\sigma=\langle\alpha \beta \gamma \delta \varepsilon\rangle$.

We will write $\tau \prec \sigma$ to mean $\tau$ is a proper face of $\sigma$, and $\tau \preccurlyeq \sigma$ when equality is also allowed.

Set $c_{\alpha}^{\sigma}=c_{\alpha} \cap \sigma$. With respect to barycentric coordinates $t_{\alpha}, \ldots, t_{\varepsilon}$ on $\sigma, c_{\alpha}^{\sigma}$ $=\left\{\left(t_{\alpha}, \ldots, t_{\varepsilon}\right): 0 \leqq t_{\beta}, t_{\gamma}, t_{\delta}, t_{\varepsilon} \leqq t_{\alpha}\right\}$. We introduce modified barycentric coordinates on $c_{\alpha}^{\sigma}$ :

$$
s_{\lambda}=t_{\lambda} / t_{\alpha} \text { for } \lambda=\beta, \gamma, \delta, \varepsilon
$$

Now let $c_{\alpha \beta}^{\sigma}=c_{\alpha} \cap c_{\beta} \cap \sigma$; this is the intersection of $\sigma$ with the domain of the transition function $v_{\alpha \beta}$ to be constructed. In modified barycentric coordinates, $c_{\alpha \beta}^{\sigma}$ is identified with the 3 -cube $\left\{\left(s_{\gamma}, s_{\delta}, s_{\varepsilon}\right): 0 \leqq s_{\gamma}, s_{\delta}, s_{\varepsilon} \leqq 1\right\}$. The faces of $c_{\alpha \beta}^{\sigma}$ are given by requiring of one or more $t_{\lambda}$ that it be either 0 or equal to $t_{\alpha}$ and $t_{\beta}$; this is equivalent to requiring that the corresponding $s_{\lambda}$ be 0 or 1 respectively. In particular the vertices of $c_{\alpha \beta}^{\sigma}$ are the barycenters of those simplexes $\tau$ such that $\langle\alpha \beta\rangle \preccurlyeq \tau \preccurlyeq \sigma$. The pairs of opposite 2-dimensional (maximal) faces of $c_{\alpha \beta}^{\sigma}$ can be described thus (see Fig. 2.2): For each $\lambda=\gamma, \delta$, $\varepsilon$, set $\tau(\lambda)=$ the face opposite $\lambda$ and set $c_{\alpha \beta \lambda}^{\sigma}=c_{\alpha \beta}^{\sigma} \cap c_{\lambda}$; then $c_{\alpha \beta}^{\tau(\lambda)}=\left\{s_{\lambda}=0\right\}$ and $c_{\alpha \beta \lambda}^{\sigma}=\left\{s_{\lambda}=1\right\}$ are opposite faces of $c_{\alpha \beta}^{\sigma}$.

Set:

$$
\begin{aligned}
& \partial^{0}\left(c_{\alpha \beta}^{\sigma}\right)=\bigcup\left\{c_{\alpha \beta}^{\tau(\lambda)}: \lambda=\gamma, \delta, \varepsilon\right\}, \\
& \partial^{1}\left(c_{\alpha \beta}^{\sigma}\right)=\bigcup\left\{c_{\alpha \beta \lambda}^{\sigma}: \lambda=\gamma, \delta, \varepsilon\right\},
\end{aligned}
$$

so $\partial c_{\alpha \beta}^{\sigma}=\partial^{0} \cup \partial^{1}$, and $\partial^{0}\left(c_{\alpha \beta}^{\sigma}\right)=c_{\alpha \beta}^{\sigma} \cap \partial \sigma$.

We will need expressions for the intersections between 2-dimensional faces of $c_{\alpha \beta}^{\sigma}$. Let $\lambda, \mu \in\{\gamma, \delta, \varepsilon\}$ be distinct, and set $\tau=\tau(\lambda), \tau^{\prime}=\tau(\mu)$. Then

$$
\begin{gathered}
c_{\alpha \beta}^{\tau} \cap c_{\alpha \beta}^{\tau^{\prime}}=c_{\alpha \beta}^{\tau^{\prime \prime}}, \text { where } \tau^{\prime \prime}=\tau \cap \tau^{\prime} ; \\
c_{\alpha \beta}^{\tau} \cap c_{\alpha \beta \mu}^{\sigma}=c_{\alpha \beta \mu}^{\tau} ; \\
c_{\alpha \beta \lambda}^{\sigma} \cap c_{\alpha \beta \mu}^{\sigma}=\left(c_{\alpha} \cap c_{\beta} \cap c_{\lambda} \cap c_{\mu}\right) \cap \sigma, \text { denoted } c_{\alpha \beta \lambda \mu}^{\sigma} .
\end{gathered}
$$




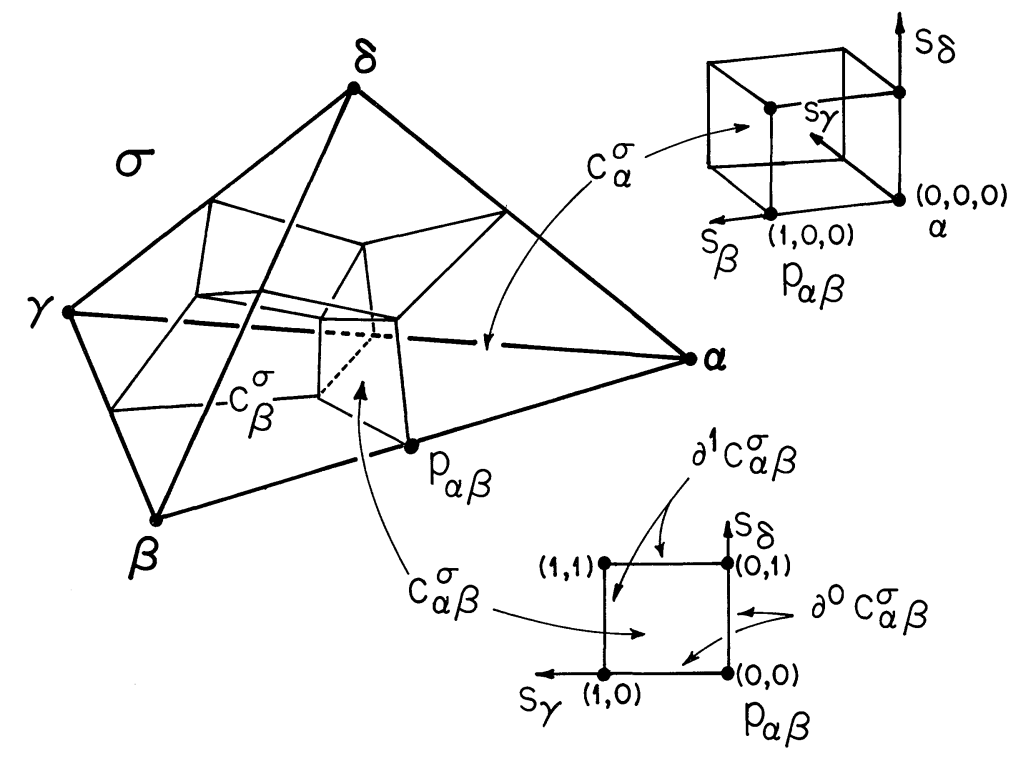

Fig. 2.2. Modified barycentric coordinates on $\sigma=\langle\alpha \beta \gamma \delta\rangle$

We will also use the linear structure on $c_{\alpha \beta}^{\sigma}$ provided by the coordinates $\left(s_{\gamma}, s_{\delta}, s_{\varepsilon}\right)$ to express $c_{\alpha \beta}^{\sigma}$ as a cone with vertex the point $p_{\alpha \beta}=(0,0,0)$, and base $\partial^{1} c_{\alpha \beta}^{\sigma}$ $=\left\{\left(s_{\gamma}, s_{\delta}, s_{\varepsilon}\right): \max s_{\lambda}=1\right\}$. We write $c_{\alpha \beta}^{\sigma}=p_{\alpha \beta} * \partial^{1} c_{\alpha \beta}^{\sigma}$.

When we speak of a geodesic $\mathfrak{g}$ in $\mathrm{SU}_{2}$ from $X$ to $Y$ we mean a path $\mathfrak{g}:[0,1]$ $\rightarrow \mathrm{SU}_{2}$ such that $\mathrm{g}(0)=X, \mathfrak{g}(1)=Y$, and $\mathfrak{g}$ is a geodesic parametrized proportionally to arc length.

2.10. Proof of Theorem 2.8. The theorem is proved by exhibiting an algorithm which goes from $\mathbf{u}$ and $\mathbf{o}$ to a family $\mathbf{v}$ of functions $v_{\alpha \beta}: c_{\alpha} \cap c_{\beta} \rightarrow \mathrm{SU}_{2}$ satisfying (2.2.1), (2.8.1) and (2.8.2). This algorithm will construct $v_{\alpha \beta}$ by piecing together functions $v_{\alpha \beta}^{\sigma}: c_{\alpha \beta}^{\sigma} \rightarrow \mathrm{SU}_{2}$, one for each triple $\sigma, \alpha, \beta$, where $\alpha$ and $\beta$ are vertices of $\sigma$.

These functions will be defined inductively on the dimension of $\sigma$, beginning with all $\sigma$ of dimension 1 .

For a fixed $\sigma$ it is enough to construct $v_{\alpha \beta}^{\sigma}$ when $\alpha$ o-precedes $\beta$. Let $n$ be the number of vertices of $\sigma$ which are o-between $\alpha$ and $\beta$.

For a fixed $\sigma$, the functions $v_{\alpha \beta}^{\sigma}$ are constructed by induction on $n$.

The domain $c_{\alpha \beta}^{\sigma}$ of $v_{\alpha \beta}^{\sigma}$ is parametrized by $\left(s_{\lambda}, \ldots, s_{\mu}, \ldots, s_{v}, \ldots\right)$, where $\lambda, \ldots$ o-precede $\alpha, \mu, \ldots$ are o-between $\alpha$ and $\beta$ (there are $n$ of these); and $v, \ldots$ o-follow $\beta$. (We will continue to use $\lambda, \mu, v$ in this sense.) Let $\varrho=\langle\alpha, \mu, \ldots, \beta\rangle$ be the face of $\sigma$ whose vertices are $\alpha, \beta$ and the $n$ vertices of $\sigma$ that are o-between $\alpha$ and $\beta$. Then $c_{\alpha \beta}^{\varrho}$ is parametrized by $\left(s_{\mu}, \ldots\right)$. Our algorithm has the following feature.

$$
\text { If } \varrho \neq \sigma, v_{\alpha \beta}^{\sigma}\left(s_{\lambda}, \ldots, s_{\mu}, \ldots, s_{v}, \ldots\right)=v_{\alpha \beta}^{\varrho}\left(s_{\mu}, \ldots\right) \text {. }
$$


This feature expresses the "as constant as possible" principle mentioned in the Introduction. In particular

$$
\begin{aligned}
& \text { When } \alpha \text { and } \beta \text { are } \mathbf{0} \text {-consecutive in } \sigma, v_{\alpha \beta}^{\sigma} \text { is constant and equal } \\
& \text { to } u_{\alpha \beta} \text {. }
\end{aligned}
$$

Finally, when $\varrho=\sigma$, so that $\alpha$ is the o-first, and $\beta$ the o-last, vertex of $\sigma$, then our algorithm will use the conical structure $c_{\alpha \beta}^{\sigma}=p_{\alpha \beta} * \partial^{1} c_{\alpha \beta}^{\sigma}$ of (2.7). For every $x \in \partial^{1} c_{\alpha \beta}^{\sigma}$, $v_{\alpha \beta}^{\sigma}$ must map the generator $\overline{p_{\alpha \beta} x}$ into the shortest geodesic $\mathfrak{g}$ in $\mathrm{SU}_{2}$ from $u_{\alpha \beta}$ $=v_{\alpha \beta}^{\sigma}\left(p_{\alpha \beta}\right)$ to $v_{\alpha \beta}^{\sigma}(x)$; more explicitly,

$$
v_{\alpha \beta}^{\sigma}\left((1-s) p_{\alpha \beta}+s x\right)=\mathfrak{g}(s) .
$$

The features (2.10.1-5) of our algorithm determine it completely. The remainder of this proof serves to check that the procedure is coherent, that it yields $v_{\alpha \beta}$ 's satisfying (2.2.1), (2.8.1) and (2.8.2), and that it can in fact be carried out (i.e. that the shortest geodesics mentioned in (2.10.5) actually exist).

Checking the coherence of the procedure means verifying

When $\langle\alpha \beta\rangle \preccurlyeq \tau \prec \sigma$, then $v_{\alpha \beta}^{\sigma} \mid c_{\alpha \beta}^{\tau}=v_{\alpha \beta}^{\tau}$.

The cocycle condition (2.2.1) becomes

$$
\begin{gathered}
v_{\alpha \beta}^{\sigma} v_{\beta \alpha}^{\sigma}=I \text { on } c_{\alpha \beta}^{\sigma}, \\
v_{\alpha \beta}^{\sigma}=v_{\alpha \mu}^{\sigma} v_{\mu \beta}^{\sigma} \text { on } c_{\alpha \mu \beta}^{\sigma} \text {, when }\langle\alpha \mu \beta\rangle \preccurlyeq \sigma .
\end{gathered}
$$

Conditions (2.8.1) and (2.8.2), describing how close $\mathbf{v}$ fits to $\mathbf{u}$, become

$$
\begin{gathered}
v_{\alpha \beta}^{\sigma}\left(p_{\alpha \beta}\right)=u_{\alpha \beta}, \\
v_{\alpha \beta}^{\sigma}(x) \cdot u_{\alpha \beta}^{-1} \in \text { convex hull }\left\{u_{\mathbf{o}}(\tau):\langle\alpha \beta\rangle \preccurlyeq \tau \preccurlyeq \sigma\right\}, \text { for all } x \in c_{\alpha \beta}^{\sigma} .
\end{gathered}
$$

2.11. Checking the Algorithm. According to (2.10.1) and (2.10.2) we must proceed by a double induction, first on $\operatorname{dim} \sigma$, and then on $\operatorname{dim} \varrho$. The initial step, $\operatorname{dim} \sigma=1$, is given by (2.10.4); in that case $v_{\alpha \beta}^{\sigma}=u_{\alpha \beta}$. When we come to define $v_{\alpha \beta}^{\sigma}$ with $\operatorname{dim} \sigma>1$, we find that this function is already defined on some (or all) of the maximal faces of $c_{\alpha \beta}^{\sigma}$ : by $v_{\alpha \beta}^{\tau}$ on $c_{\alpha \beta}^{\tau}$ for $\langle\alpha \beta\rangle \preccurlyeq \tau \prec \sigma$ and $\operatorname{dim} \tau=\operatorname{dim} \sigma-1$ [see (2.10.6)]; and by $v_{\alpha \mu}^{\sigma} v_{\mu \beta}^{\sigma}$ on $c_{\alpha \mu \beta}^{\sigma}$ for $\mu$ o-between $\alpha$ and $\beta$ [see (2.10.8)].

2.12. Lemma. These functions agree on common intersections. Specifically,

$$
\begin{array}{ccc}
v_{\alpha \beta}^{\tau}=v_{\alpha \beta}^{\tau^{\prime}} \quad \text { on } & c_{\alpha \beta}^{\tau} \cap c_{\alpha \beta}^{\tau^{\prime}} ; \\
v_{\alpha \beta}^{\tau}=v_{\alpha \mu}^{\sigma} v_{\mu \beta}^{\sigma} \quad \text { on } & c_{\alpha \beta}^{\tau} \cap c_{\alpha \mu \beta}^{\sigma} ; \\
v_{\alpha \mu}^{\sigma} v_{\mu \beta}^{\sigma}=v_{\alpha \mu^{\prime}}^{\sigma} v_{\mu^{\prime} \beta}^{\sigma} & \text { on } & c_{\alpha \mu \beta}^{\sigma} \cap c_{\alpha \mu^{\prime} \beta}^{\sigma} .
\end{array}
$$

In case $\varrho \neq \sigma$ and we define $v_{\alpha \beta}^{\sigma}$ by (2.10.3), we must check that this is compatible with the way $v_{\alpha \beta}^{\sigma}$ was prescribed above.

2.13. Lemma. When $\varrho \neq \sigma$ :

$$
\begin{gathered}
v_{\alpha \beta}^{\sigma}=v_{\alpha \beta}^{\tau} \quad \text { on } \quad c_{\alpha \beta}^{\tau} ; \\
v_{\alpha \beta}^{\sigma}=v_{\alpha \mu}^{\sigma} v_{\mu \beta}^{\sigma} \quad \text { on } \quad c_{\alpha \mu \beta}^{\sigma} .
\end{gathered}
$$

In case $\varrho=\sigma$ the only compatibility requirement is: 
2.14. Lemma. When $\varrho=\sigma, v_{\alpha \beta}^{\sigma}=v_{\alpha \beta}^{\tau}$ on $c_{\alpha \beta}^{\tau}$.

2.15. Finally, in order to apply (2.10.5) we must verify that there is indeed a unique shortest geodesic from $u_{\alpha \beta}$ to $v_{\alpha \beta}^{\sigma}(x)$ for every $x$ in $\partial^{1} c_{\alpha \beta}^{\sigma}$; in other words, that $u_{\alpha \beta}$ and $v_{\alpha \beta}^{\sigma}(x)$ are never antipodal points of $S^{3}$.

2.16. Lemmas 2.12-2.14 can be straightforwardly proved by an induction argument following (2.10.1) and (2.10.2). What we shall do now is run through the inductive construction of $\mathbf{v}$ from the beginning, concentrating on the general position requirement just mentioned, and on checking (2.10.10).

The construction of the $v_{\alpha \beta}^{\sigma}$ 's starts with

2.17. $\operatorname{Dim} \sigma=1$. This case is covered by (2.10.4): $c_{\alpha \beta}^{\sigma}$ is simply the barycenter $p_{\alpha \beta}$ of $\langle\alpha \beta\rangle$, and $v_{\alpha \beta}^{\sigma}\left(p_{\alpha \beta}\right)=u_{\alpha \beta}$.

2.18. $\operatorname{Dim} \sigma=2$. We are assuming that every 2-simplex is a face of a 4-simplex, say $\langle 01234\rangle$ with its vertices so o-ordered. For example, $\sigma=\langle 012\rangle$. We first consider the cases $\alpha=0, \beta=1$ and $\alpha=1, \beta=2$, following (2.10.2). In the first of these cases $\varrho=\langle 01\rangle$, and (2.10.4) applies:

$$
v_{01}^{\sigma}=v_{01}^{\varrho}=u_{01} \text { is the constant map. }
$$

Similarly $v_{12}^{\sigma}=u_{12}$ is constant, too. We are left with the case $\alpha=0, \beta=2$; here we must apply (2.10.5). The domain of $v_{02}^{\sigma}$ is the 1 -cube $c_{02}^{\sigma}$, parametrized by $s_{1}$. Its boundary consists of two points: $s_{1}=0$ at $p=p_{02}=c_{02}^{\tau}$, the barycenter of $\tau=\langle 02\rangle$; and $s_{1}=1$ at $q=c_{021}^{\sigma}$, the barycenter of $\sigma$ (see Fig. 2.3).
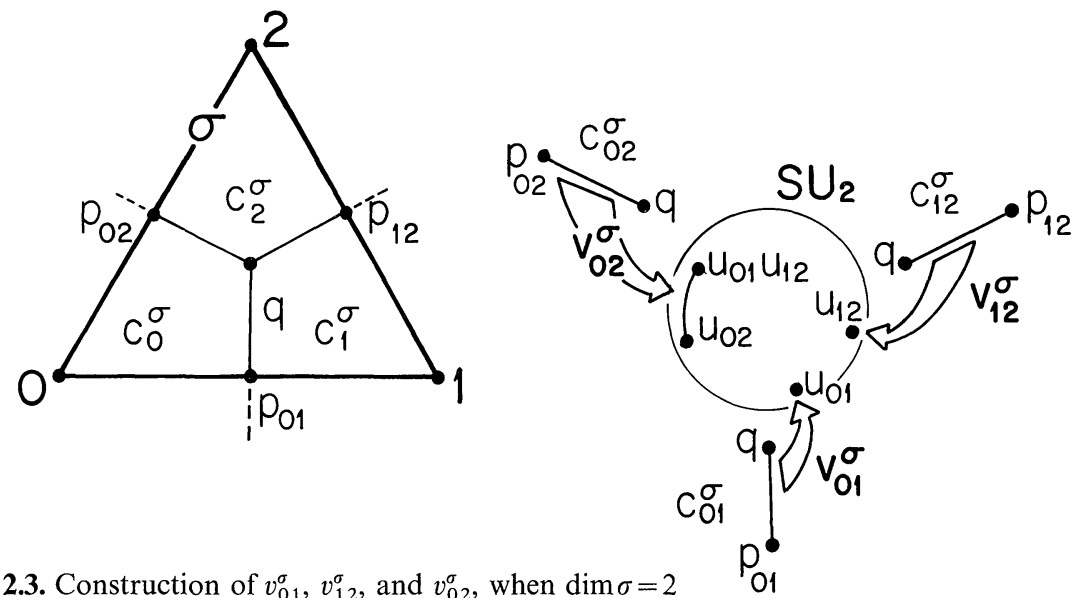

Fig. 2.3. Construction of $v_{01}^{\sigma}, v_{12}^{\sigma}$, and $v_{02}^{\sigma}$, when $\operatorname{dim} \sigma=2$

Now

$$
\begin{aligned}
v_{02}^{\sigma}(p) & =v_{02}^{\tau}(p) \quad \text { by }(2.10 .6) \\
& =u_{02} ;
\end{aligned}
$$


while

$$
\begin{aligned}
v_{02}^{\sigma}(q) & =v_{01}^{\sigma}(q) v_{12}^{\sigma}(q) \quad \text { by }(2.10 .8) \\
& =u_{01} u_{12}=u_{012} .
\end{aligned}
$$

We now need to know that (*) there is a unique shortest geodesic $\mathfrak{g}_{012}$ from $u_{02}$ to $u_{012}$. The isometry, right multiplication by $u_{20}$, matches these points with $I$ and $u_{0120}$; either Condition $A(\mathbf{o})$ [line (1)] or Condition $B(\mathbf{o})$ shows that these two points cannot be antipodal; the existence of $\mathfrak{g}_{012}$ follows. Finally, (2.10.9) and (2.10.10) are immediate from the construction. [For the other 2 -faces of $\langle 01234\rangle$, similar arguments reduce the analogues of $(*)$ to $A(\mathbf{0})$ and $B(\mathbf{0})$.]

2.19. $\operatorname{Dim} \sigma=3$. Again, we assume every 3-simplex is a face of a 4-simplex, say $\langle 01234\rangle$. For example, $\sigma=\langle 0123\rangle$. We start with $v_{01}^{\sigma}$, where $\varrho=\langle 01\rangle$ has dimension 1. By (2.10.4), $v_{01}^{\sigma}\left(s_{2}, s_{3}\right)=u_{01}$ is constant, and so are $v_{12}^{\sigma}=u_{12}$ and $v_{23}^{\sigma}$ $=u_{23}$.

Now consider $v_{02}^{\sigma}$, where $\varrho=\langle 012\rangle$ is 2-dimensional. By $(2.10 .3), v_{02}^{\sigma}\left(s_{1}, s_{3}\right)$ $=v_{02}^{\varrho}\left(s_{1}\right)=\mathfrak{g}_{012}\left(s_{1}\right)$. Similarly, $v_{13}^{\sigma}\left(s_{0}, s_{2}\right)=\mathfrak{g}_{123}\left(s_{2}\right)$.

It remains to define $v_{03}^{\sigma}\left(s_{1}, s_{2}\right)$. Set $\tau=\langle 013\rangle, \tau^{\prime}=\langle 023\rangle$. Then, by (2.10.6) and (2.10.8), $v_{03}^{\sigma}$ is determined on $\partial c_{03}^{\sigma}$ :

$$
\begin{aligned}
v_{03}^{\sigma}\left(s_{1}, 0\right) & =v_{03}^{\tau}\left(s_{1}\right) \\
& =\mathfrak{g}_{013}\left(s_{1}\right), \text { on } c_{03}^{\tau} ; \\
v_{03}^{\sigma}\left(0, s_{2}\right) & =v_{03}^{\tau^{\prime}}\left(s_{2}\right) \\
& =\mathfrak{g}_{023}\left(s_{2}\right), \text { on } c_{03}^{\tau^{\prime}} ; \\
v_{03}^{\sigma}\left(s_{1}, 1\right) & =v_{02}^{\sigma}\left(s_{1}\right) v_{23}^{\sigma}(1) \\
& =\mathfrak{g}_{012}\left(s_{1}\right) u_{23}, \text { on } c_{032}^{\sigma} ; \\
v_{03}^{\sigma}\left(1, s_{2}\right) & =v_{01}^{\sigma}(1) v_{13}^{\sigma}\left(s_{2}\right) \\
& =u_{01} \mathfrak{g}_{123}\left(s_{2}\right), \text { on } c_{031}^{\sigma} .
\end{aligned}
$$

It follows from Lemma 2.12 that these maps, defined on the 1 -faces of $c_{03}^{\sigma}$, do indeed agree at its vertices; in fact

$$
\begin{aligned}
& v_{03}^{\sigma}(0,0)=u_{03}, \\
& v_{03}^{\sigma}(1,0)=u_{013}, \\
& v_{03}^{\sigma}(0,1)=u_{023}, \\
& v_{03}^{\sigma}(1,1)=u_{0123} .
\end{aligned}
$$

To extend $v_{03}^{\sigma}$ over $c_{03}^{\sigma}$ we are required by (2.10.5) to regard $c_{03}^{\sigma}$ as a cone from $p_{03}$ (where $s_{1}=s_{2}=0$ ) on $\partial^{1} c_{03}^{\sigma}$ (where $s_{1}=1$ or $s_{2}=1$ ), and to map generators into shortest geodesics from $v_{03}^{\sigma}\left(p_{03}\right)=u_{03}$ to points of $v_{03}^{\sigma}\left(\partial^{1} c_{03}^{\sigma}\right)$. It must therefore be shown that $(* *) u_{03}$ is not antipodal to any point of $v_{03}^{\sigma}\left(\partial^{1} c_{03}^{\sigma}\right)$. Now $\partial^{1} c_{03}^{\sigma}$ consists of two 1-cells, $c_{031}^{\sigma}$ (where $s_{1}=1$ ) and $c_{032}^{\sigma}$ (where $s_{2}=1$ ). We shall show that $u_{03}$ is not antipodal to any point of $v_{03}^{\sigma}\left(c_{031}^{\sigma}\right)$; the argument for $c_{032}^{\sigma}$ is completely analogous. Under $v_{03}^{\sigma}, c_{031}^{\sigma}$ is mapped into the geodesic $u_{01} \mathfrak{g}_{123}$ from $u_{01} u_{13}=u_{013}$ to $u_{01} u_{123}=u_{0123}$. Right multiplication by $u_{340}$ takes the three points $u_{03}, u_{013}$, $u_{0123}$ to $u_{0340}, u_{01340}, u_{012340}$. By Condition $A(\mathbf{o})$ (line 4$)$ these last three points, 


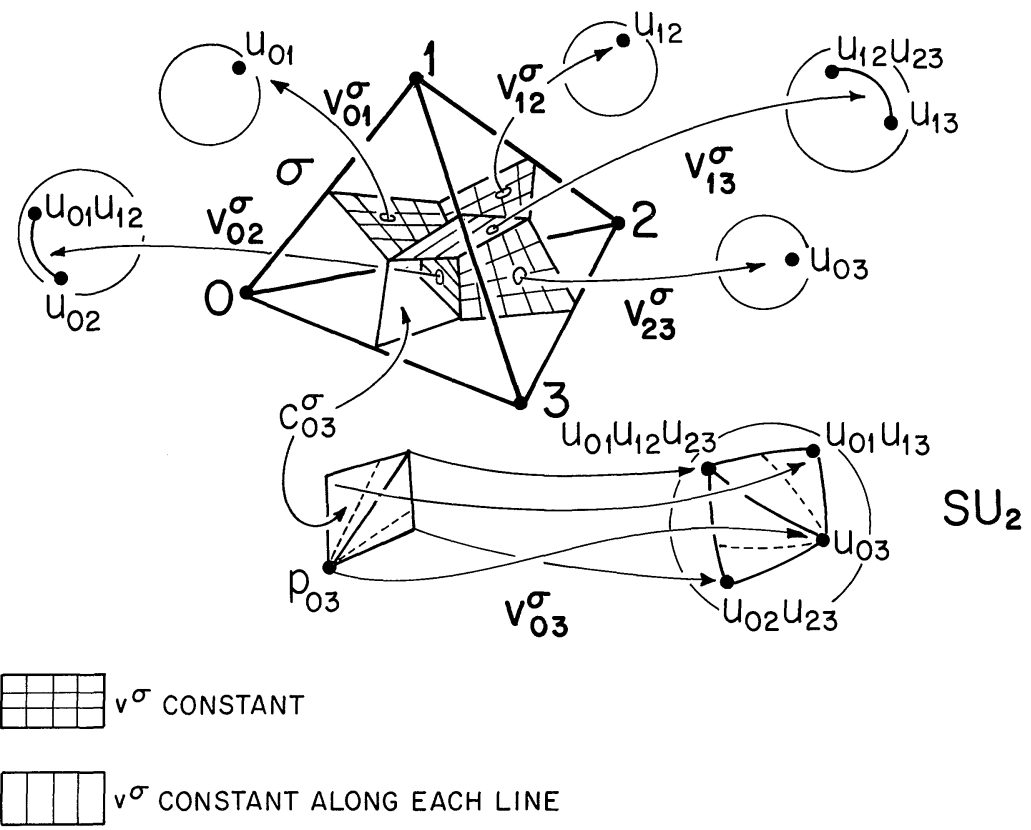

Fig. 2.4. Construction of $v_{03}^{\sigma}$, for $\sigma=\langle 0123\rangle$

and therefore the first three, are linearly independent in $\mathbf{R}^{4}$; but if the great circle through $u_{013}$ and $u_{0123}$ contained $-u_{03}$ it would also contain $u_{03}$, so the first three points would lie in a single plane through the origin, contradicting linear independence. Otherwise Condition $B(\mathbf{o})$ implies that $u_{03}, u_{013}$, and $u_{0123}$ lie in an open ball of radius $\pi / 2$ in $\mathrm{SU}_{2}=S^{3}$; and such balls are strictly convex. In either case, we obtain the desired result. [For the other 3-faces of $\langle 01234\rangle$, similar arguments reduce the analogues of $(* *)$ to $A(\mathbf{o})$ and $B(\mathbf{0})$.].

We may therefore define $v_{03}^{\sigma}$ by (2.10.5). We shall also use the notation $\mathfrak{h}_{0123}\left(s_{1}, s_{2}\right)$ for this map. Its image (see Fig. 2.4) is the union of two geodesic triangles in $S^{3}$. As before, (2.10.9) and (2.10.10) are immediate from the construction.

2.20. $\operatorname{Dim} \sigma=4$. Say $\sigma=\langle 01234\rangle$. Applying (2.10.3) and (2.10.4) as before, we obtain the following.

$$
v_{01}^{\sigma}=u_{01}, \quad v_{12}^{\sigma}=u_{12}, \quad v_{23}^{\sigma}=u_{23}, \quad v_{34}^{\sigma}=u_{34}
$$

are constant maps.

$$
\begin{aligned}
& v_{02}^{\sigma}\left(s_{1}, s_{3}, s_{4}\right)=\mathfrak{g}_{012}\left(s_{1}\right), \\
& v_{13}^{\sigma}\left(s_{0}, s_{2}, s_{4}\right)=\mathfrak{g}_{123}\left(s_{2}\right), \\
& v_{24}^{\sigma}\left(s_{0}, s_{1}, s_{3}\right)=\mathfrak{g}_{234}\left(s_{3}\right), \\
& v_{03}^{\sigma}\left(s_{1}, s_{2}, s_{4}\right)=\mathfrak{h}_{0123}\left(s_{1}, s_{2}\right), \\
& v_{14}^{\sigma}\left(s_{0}, s_{2}, s_{3}\right)=\mathfrak{h}_{1234}\left(s_{2}, s_{3}\right) .
\end{aligned}
$$


We turn finally to the definition of $v_{04}^{\sigma}\left(s_{1}, s_{2}, s_{3}\right)$. On $\partial c_{04}^{\sigma}, v_{04}^{\sigma}$ is already prescribed:

$$
\begin{aligned}
& \text { on } \partial^{0} c_{04}^{\sigma} \text { by } \\
& v_{04}^{\sigma}\left(0, s_{2}, s_{3}\right)=\mathfrak{h}_{0234}\left(s_{2}, s_{3}\right), \\
& v_{04}^{\sigma}\left(s_{1}, 0, s_{3}\right)=\mathfrak{h}_{0134}\left(s_{1}, s_{3}\right), \\
& v_{04}^{\sigma}\left(s_{1}, s_{2}, 0\right)=\mathfrak{h}_{0124}\left(s_{1}, s_{2}\right) ; \\
& \text { on } \partial^{1} c_{04}^{\sigma} \text { by } \\
& v_{04}^{\sigma}\left(1, s_{2}, s_{3}\right)=v_{01}^{\sigma} v_{14}^{\sigma} \\
&=u_{01} \mathfrak{h}_{1234}\left(s_{2}, s_{3}\right), \text { on } c_{041}^{\sigma}, \\
& v_{04}^{\sigma}\left(s_{1}, 1, s_{3}\right)=v_{02}^{\sigma} v_{24}^{\sigma} \\
&= \mathfrak{g}_{012}\left(s_{1}\right) \mathfrak{g}_{234}\left(s_{3}\right), \text { on } c_{042}^{\sigma}, \\
& v_{04}^{\sigma}\left(s_{1}, s_{2}, 1\right)=v_{03}^{\sigma} v_{34}^{\sigma} \\
&=\mathfrak{h}_{0123}\left(s_{1}, s_{2}\right) u_{34}, \text { on } c_{043}^{\sigma} .
\end{aligned}
$$

(To show these are compatible, we appeal to Lemma 2.12.)

To extend $v_{04}^{\sigma}$ over $c_{04}^{\sigma}$ according to (2.10.5) we must join $v_{04}^{\sigma}(0,0,0)=u_{04}$ to every point of $v_{04}^{\sigma}\left(\partial^{1} c_{04}^{\sigma}\right)$ by a unique shortest geodesic; so we must show that no such point is antipodal to $u_{04}$. Now $v_{04}^{\sigma}\left(\partial^{1} c_{04}^{\sigma}\right)$ consists of four geodesic triangles and a quadrilateral, doubly ruled surface.

A typical one of the geodesic triangles has vertices $u_{014}, u_{0124}$ and $u_{01234}$ [this one is half of $\left.v_{04}^{\sigma}\left(c_{041}^{\sigma}\right)\right]$. By Condition $A(\mathbf{o})$ (line 1) these three points together with $u_{04}$ are all in general position; by Condition $B(\mathbf{0})$ all four lie in an open $\pi / 2$-ball. In either case it follows that there is, as required, a unique shortest geodesic from $u_{04}$ to every point of the geodesic triangle. Thus we can carry out the construction of (2.10.5) to extend $v_{04}^{\sigma}$ over the join of $p_{04}=(0,0,0)$ to the half of $c_{041}^{\sigma}$ under discussion. This argument shows how $v_{04}^{\sigma}$ can be defined on the entire cone from $p_{04}$ on $c_{041}^{\sigma} \cup c_{043}^{\sigma}$.

2.22. It remains to be verified that there is a unique shortest geodesic from $u_{04}$ to each point of $v_{04}^{\sigma}\left(c_{042}^{\sigma}\right)$.

Case 1. $\sigma$ satisfies $B(\mathbf{o})$. The vertices of $v_{04}^{\sigma}\left(c_{042}^{\sigma}\right)$ are $u_{024}, u_{0124}, u_{0234}$, and $u_{01234}$. These all lie in the open $\pi / 2$-ball $B$ about $u_{04}$; for if we multiply the four vertices by $u_{40}$ on the right we obtain four "increasing" loop-products, which by $B(\mathbf{0})$ are all within $\pi / 2$ of $I$. Since $B$ is geodesically convex, the geodesics $g_{012} g_{234}(0)$ from $u_{024}$ to $u_{0124}$ and $\mathfrak{g}_{012} \mathfrak{g}_{234}(1)$ from $u_{0234}$ to $u_{01234}$ both lie in $B$. That is, for any $s_{1}$, $\mathfrak{g}_{012}\left(s_{1}\right) \mathfrak{g}_{234}(0)$ and $\mathfrak{g}_{012}\left(s_{1}\right) \mathfrak{g}_{234}(1)$ are in $B$. Hence the geodesic $\mathfrak{g}_{012}\left(s_{1}\right) \mathfrak{g}_{234}$ between these two points also lies in $B$. In particular, for any $s_{1}$ and $s_{3}$, the point $\mathfrak{g}_{012}\left(s_{1}\right) \mathfrak{g}_{234}\left(s_{3}\right)$ is in $B$; and therefore there is a unique shortest geodesic to it from $u_{04}$, as required.

Case 2. $\sigma$ satisfies $A(\mathbf{o})$. Suppose $u_{04}$ were antipodal to some point of $v_{04}^{\sigma}\left(c_{042}^{\sigma}\right)$. Then we should have

$$
\mathfrak{g}_{012}\left(s_{1}\right) \mathfrak{g}_{234}\left(s_{3}\right)=-u_{04}, \text { for some } s_{1} \text { and } s_{3} .
$$




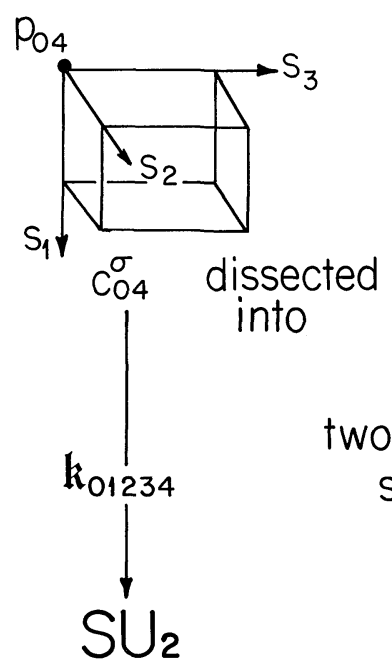

Fig. 2.5. Geometry of the map $\mathfrak{f}_{01234}$
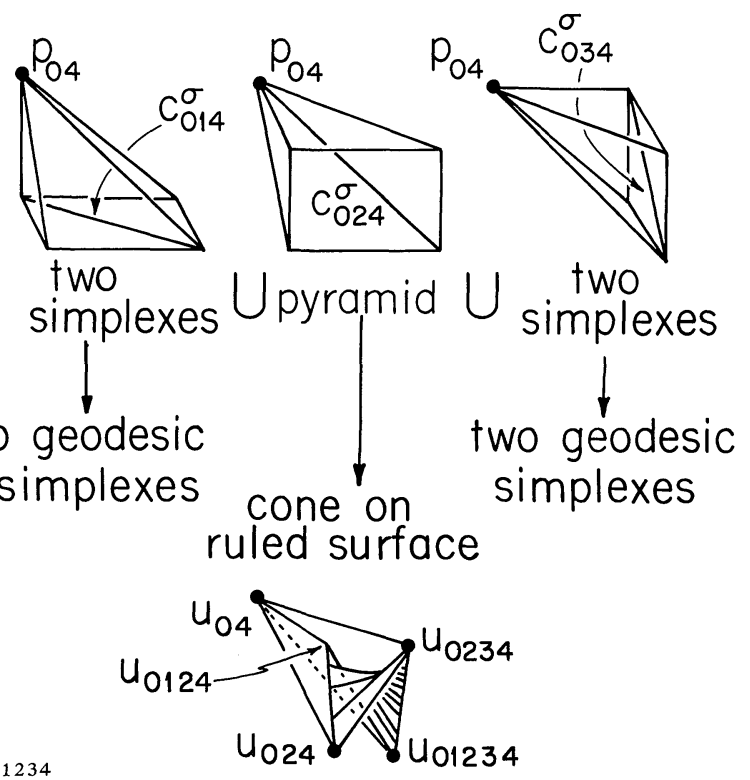

We rewrite this as

$$
\mathfrak{g}_{234}\left(s_{3}\right) u_{40}=-\left(\mathfrak{g}_{012}\left(s_{1}\right)\right)^{-1} \text {. }
$$

Now $\mathfrak{g}_{234} u_{40}$ is a segment of the geodesic circle $S$ through $u_{24} u_{40}=u_{240}$ and $u_{234} u_{40}=u_{2340}$, while $\left(g_{012}\right)^{-1}$ is part of the geodesic circle $S^{\prime}$ through $u_{02}^{-1}=u_{20}$ and $u_{012}^{-1}=u_{210}$. We are thus supposing that $S$ contains a point antipodal to some point of $S^{\prime}$; this implies that $S$ and $S^{\prime}$ together lie on some geodesic 2-sphere in $S^{3}$. That 2-sphere, which is the intersection of $S^{3}$ with a 3-plane through the origin in $\mathbf{R}^{4}$, must contain the points $u_{240}, u_{2340}, u_{20}$, and $u_{210}$, which thus have to be linearly dependent. But if we multiply these four points on the left by $u_{012}$ we obtain $u_{01240}, u_{012340}, u_{0120}$, and $I$, which are independent by Condition $A(\mathbf{0})$, line 1. This is a contradiction.

So in either case there is a unique shortest geodesic from $u_{04}$ to every point of $v_{04}^{\sigma}\left(c_{042}^{\sigma}\right)$, as required.

We will use the symbol $\mathfrak{f}_{01234}$ for the extension of $v_{04}^{\sigma}$ to all of $c_{04}^{\sigma}$. Again, (2.10.9) and (2.10.10) are immediate consequences of the construction. This completes the proof of Theorem 2.8 .

For use in Sect. 4, we remark that implicit in the definition of $\mathfrak{f}_{01234}$ is a partition of the cube $c_{04}^{\sigma}$ into four simplexes and a square-based pyramid (see Fig. 2.5). Each simplex is sent by $\mathfrak{f}_{01234}$ to a spherical simplex in $S^{3}$, and the pyramid to the geodesic cone on a quadrilateral, doubly ruled surface.

\section{The Uniqueness Problem}

3.1. The last section presented an algorithm (Theorem 2.8) which, given an $\mathrm{SU}_{2}$ valued lattice gauge field $\mathbf{u}$ defined on a 4-dimensional simplicial complex $\Lambda$, 
together with a local ordering $\mathbf{0}$ of the vertices of $\Lambda$, produces a coordinate $\mathrm{SU}_{2}$-bundle $\mathbf{v}$ with trivializing sets the 4-cells dual to the vertices of $\Lambda$. [u must not belong to the exceptional set $K(\mathbf{o})$.]

The next section will give an extension of this algorithm, going from $\mathbf{v}$ to the second Chern number $C_{2}(\xi)$ of the underlying principal bundle $\xi$. We would like to think of this number as "the topological charge of $\mathbf{u}$," but first we must ask to what extent the number $C_{2}(\xi)$ really does depend only on $\mathbf{u}$. That is the problem addressed in this section. We will work on the uniqueness of $\xi$, an equivalent problem in this context.

3.2. The coordinate bundle given by the algorithm satisfies the equation

$$
v_{\alpha \beta}\left(p_{\alpha \beta}\right)=u_{\alpha \beta},
$$

where $p_{\alpha \beta}$ is the center of the common face of the dual cells $c_{\alpha}$ and $c_{\beta}$; so the construction can be thought of as stretching the transporter $u_{\alpha \beta}$ over the face $c_{\alpha} \cap c_{\beta}$ so as to satisfy the cocycle condition at the edges. It is fairly clear, however, that unless the stretching is controlled, the topological type of the underlying principal bundle $\xi$ may be quite arbitrary; the control we will use comes from placing bounds on the function $d\left(v_{\alpha \beta}(x), u_{\alpha \beta}\right), x \in c_{\alpha} \cap c_{\beta}$. Working back to the lattice gauge field we can prove uniqueness results, which can be summarized as follows.

In the space $\mathbf{G}$ of all $\mathrm{SU}_{2}$-valued lattice gauge fields on $\Lambda$ there is the subset $F$ of flat fields: those giving transporter product $=I$ around any plaquette, and there are three increasing open sets containing $F: F \subset A_{1} \subset A_{2} \subset A_{3} \subset \mathbf{G}$ with the following properties. If $\mathbf{u} \in A_{1}$, then it has a "best approximation" $\mathbf{v}$ in the sense that any $\mathbf{v}^{\prime}$ as close to $\mathbf{u}$ as $\mathbf{v}$ is will define an isomorphic principal bundle; this $\mathbf{v}$ is produced by our algorithm. If $\mathbf{u} \in A_{2}$, then the principal bundle determined by applying our algorithm to $\mathbf{u}$ does not depend on the local vertex ordering employed. If $\mathbf{u} \in A_{3}$, then the algorithm will produce a coordinate bundle when applied to $\mathbf{u}$ and a local vertex ordering $\mathbf{o}$, although different orderings may give different topological types; see Example (3.20). The complement of $A_{3}$ is the union of the sets $K(\mathbf{0})$, where $\mathbf{o}$ runs over the set of all local vertex orderings, and is therefore a set of measure zero.

3.3. Definitions of $A_{1}, A_{2}$, and $A_{3}$. The sets $K(\mathbf{o})$ were defined in Sect. 2, and $A_{3}$ is the intersection of their complements in $\mathbf{G}$. So $A_{3}$ consists of those $\mathbf{u}$ such that for every 4-simplex, and for every ordering, the continuity hypothesis [either $A(\mathbf{o})$ or $B(\mathbf{o})]$ holds.

For a simplex $\tau$, let $\tau=\langle\alpha \beta \gamma \ldots \zeta\rangle$ be any ordering of its vertices, and set $u_{\alpha \beta \gamma \ldots \zeta \alpha}$ $=u_{\alpha \beta} u_{\beta \gamma} \ldots u_{\zeta \alpha}$ as usual. The set $A_{2}$ consists of those $\mathbf{u}$ such that

$$
d\left(I, u_{\alpha \beta \gamma \ldots \zeta \alpha}\right)<\pi / 2
$$

for every $\tau=\langle\alpha \beta \gamma \ldots \zeta\rangle$ and for every ordering of the vertices of $\tau$. In other words, $B(\mathbf{o})$ holds for every 4-simplex in every local ordering $\mathbf{o}$.

Finally, let $A_{1}$ be the set of those $\mathbf{u}$ such that, for some ordering $\mathbf{0}$,

$$
d\left(I, u_{\mathbf{o}}(\tau)\right)<\pi / 8 \text { for every simplex } \tau .
$$

This is condition $B(\mathbf{o})$, except that the right-hand side has been reduced from $\pi / 2$ to $\pi / 8$. Furthermore, when $\tau$ is a 2-simplex, condition (3.3.2) is independent of $\mathbf{o}$ (this is 
easy to check). On the other hand for any $\tau$ in $\Lambda$ and any ordering $\alpha<\beta<\gamma<\ldots<\zeta$ of its vertices, the element $u_{\alpha \beta \gamma \ldots \zeta \alpha}$ can be decomposed into a product of at most three terms of the form $u_{\alpha \beta \gamma \alpha}$. This argument shows that (3.3.2) implies (3.3.1), so $A_{1} \subset A_{2}$.

3.4. Proposition. Suppose given an $\mathrm{SU}_{2}$-valued lattice gauge field $\mathbf{u}$ and an $\mathrm{SU}_{2^{-}}$ coordinate bundle $\mathbf{v}$, both defined on a 4-dimensional simplicial complex $\Lambda$, such that for every pair $\alpha, \beta$ of adjacent vertices.

$$
d\left(v_{\alpha \beta}(x), u_{\alpha \beta}\right)<\pi / 8 \quad \text { for every } x \in c_{\alpha} \cap c_{\beta} .
$$

Suppose $\mathbf{v}^{\prime}$ is another coordinate bundle on $\Lambda$, also satisfying (3.4.1). Then the principal bundles $\xi$ and $\xi^{\prime}$ determined by $\mathbf{v}$ and $\mathbf{v}^{\prime}$ respectively are isomorphic.

3.5. Note that Proposition 3.4 applies in particular when $\mathbf{u}$ is in $A_{1}$; for then the algorithm of Theorem 2.8 gives us a coordinate bundle $\mathbf{v}$ which satisfies the hypotheses of the proposition.

3.6. Proposition. Given a 4-dimensional simplicial complex $\Lambda$, a lattice gauge field $\mathbf{u}$ on $\Lambda$, belonging to $A_{2}$, and two local orderings $\mathbf{0}$ and $\mathbf{o}^{\prime}$ of the vertices of $\Lambda$, let $\mathbf{v}$ and $\mathbf{v}^{\prime}$ be the corresponding coordinate bundles constructed, according to Theorem 2.8, by our algorithm. Then the principal bundles $\xi$ and $\xi^{\prime}$ determined by $\mathbf{v}$ and $\mathbf{v}^{\prime}$ respectively are isomorphic.

3.7. The proofs of these two propositions share a common strategy. In both cases we shall construct a coordinate bundle $\mathbf{v}^{*}$ on $\Lambda \times[0,1]$ which extends $\mathbf{v}$ on $A \times\{0\}$ and $\mathbf{v}^{\prime}$ on $\Lambda \times\{1\}$. Once this is done, $\mathbf{v}^{*}$ will determine a principal bundle $\xi^{*}$ over $\Lambda \times I$ which restricts to $\xi$ over $\Lambda \times\{0\}$ and to $\xi^{\prime}$ over $\Lambda \times\{1\}$. It is then standard that $\xi$ and $\xi^{\prime}$ are isomorphic (see, for example, [27]).

In the proof of Proposition 3.6, since we shall be working in the context of our algorithm of Theorem 2.8 , it will be possible to construct $\mathbf{v}^{*}$ simply by extending that algorithm. The proof of Proposition 3.4 requires an analogous but different algorithm.

3.8. Proof of Proposition 3.4. We will abbreviate the notation for the vertices of $\Lambda \times[0,1]$ to $\alpha=(\alpha, 0)$ and $\alpha^{\prime}=(\alpha, 1)$. The 5-cells dual to these vertices (see Fig. 3.1) are $c_{\alpha}^{*}=c_{\alpha} \times[0,1 / 2]$ and $c_{\alpha^{\prime}}^{*}=c_{\alpha} \times[1 / 2,1]$. Their 4-dimensional pairwise intersections are

$$
\begin{gathered}
c_{\alpha \beta}^{*}=c_{\alpha}^{*} \cap c_{\beta}^{*}=c_{\alpha \beta} \times[0,1 / 2] \text { and } \\
c_{\alpha^{\prime} \beta^{\prime}}^{*}=c_{\alpha \beta} \times[1 / 2,1] \text { for every }\langle\alpha \beta\rangle \text { in } \Lambda ; \\
c_{\alpha \alpha^{\prime}}^{*}=c_{\alpha} \times\{1 / 2\} \text { for every vertex } \alpha \text { of } \Lambda .
\end{gathered}
$$

These are the domains of the $\mathrm{SU}_{2}$-valued functions $v_{\alpha \beta}^{*}$, $v_{\alpha^{\prime} \beta^{\prime}}^{*}$ and $v_{\alpha \alpha^{\prime}}^{*}$ to be constructed.

We define $v_{\alpha \beta}^{*}$ and $v_{\alpha^{\prime} \beta^{\prime}}^{*}$, for every $\langle\alpha \beta\rangle$, by

$$
v_{\alpha \beta}^{*}(x, t)=v_{\alpha \beta}(x), \quad v_{\alpha^{\prime} \beta^{\prime}}^{*}\left(x, t^{\prime}\right)=v_{\alpha \beta}^{\prime}(x)
$$

for every $x \in c_{\alpha \beta}, t \in[0,1 / 2], t^{\prime} \in[1 / 2,1]$. 

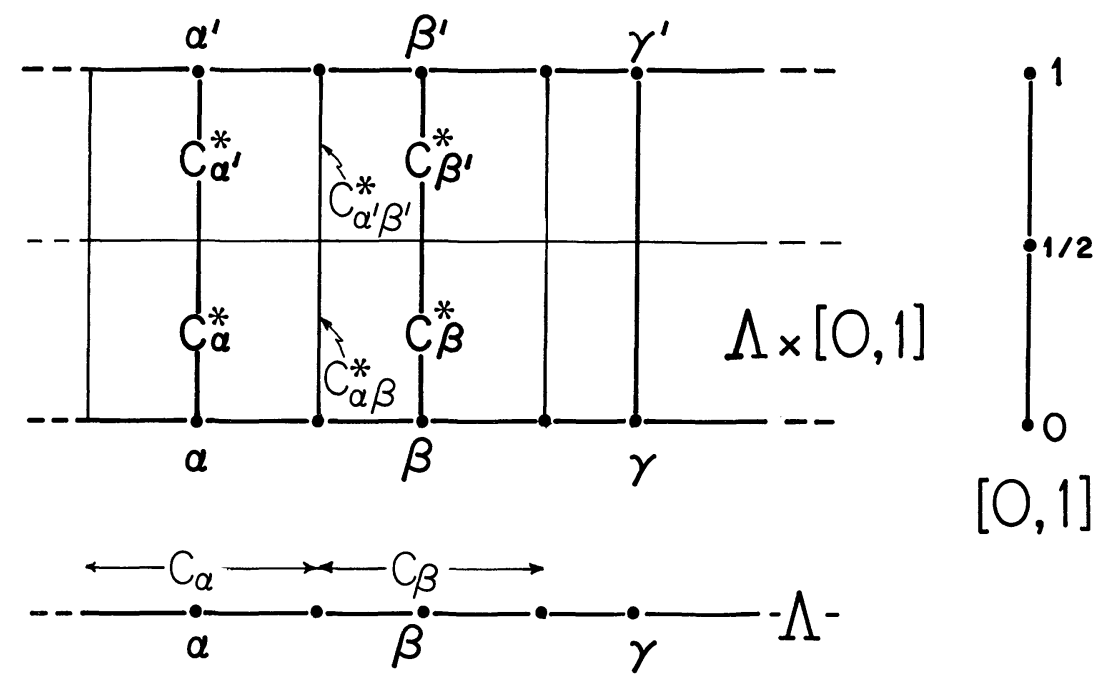

Fig. 3.1. The cell complex $\Lambda \times[0,1]$ and its dual

The definition of $v_{\alpha}=v_{\alpha \alpha^{\prime}}^{*}: c_{\alpha} \rightarrow \mathrm{SU}_{2}$ (identifying $c_{\alpha}^{*} \cap c_{\alpha^{\prime}}^{*}$ with $c_{\alpha}$ ) is made following the same principles as the definition of the $v_{\alpha \beta}$ 's in (2.8). In order to use induction we pick, once and for all, a local ordering $\mathbf{o}$ of the vertices of $\Lambda$. To support the analogy with the proof of Theorem 2.8, we may also introduce the lattice gauge field $\mathbf{u}^{*}$ on $\Lambda \times[0,1]$ defined by

$$
\begin{array}{cc}
u_{\alpha \beta}^{*}=u_{\alpha^{\prime} \beta^{\prime}}^{*}=u_{\alpha \beta} & \text { for every }\langle\alpha \beta\rangle \text { in } \Lambda, \text { and } \\
u_{\alpha \alpha^{\prime}}^{*}=I & \text { for every vertex } \alpha .
\end{array}
$$

3.9. For any simplex containing $\alpha$, let $c_{\alpha}^{\sigma}=c_{\alpha} \cap \sigma$. In parallel with (2.10.1) and (2.10.2), we define $v_{\alpha}^{\sigma}: c_{\alpha}^{\sigma} \rightarrow \mathrm{SU}_{2}$ by a double induction, first on $\operatorname{dim} \sigma$, then in each $\sigma$ proceeding from the lowest-ordered vertex to the highest. Each $c_{\alpha}^{\sigma}$ is an affine cube (see Fig. 2.2), with modified barycentric coordinates $\left(s_{\lambda}, \ldots, s_{\mu}, \ldots\right)$, where $\lambda, \ldots$ o-precede $\alpha$, and $\mu, \ldots$ o-follow $\alpha$. Let $\varrho=\langle\lambda, \ldots, \alpha\rangle$. Then in parallel with $(2.10 .3)$ and (2.10.4) we will set $v_{\alpha}^{\sigma}\left(s_{\lambda}, \ldots, s_{\mu}, \ldots\right)=v_{\alpha}^{\varrho}\left(s_{\lambda}, \ldots\right)$, and $v_{\alpha}(\alpha)=I$. Finally, when $\varrho=\sigma$, so that $\alpha$ is the o-last vertex of $\sigma$, we use the conical structure $c_{\alpha}^{\sigma}=\alpha * \partial^{1} c_{\alpha}^{\sigma}$, where $\partial^{1} c_{\alpha}^{\sigma}$ is the set on which at least one of the modified barycentric coordinates is equal to 1 . The values of the function $v_{\alpha}^{\sigma}$ will already be determined by the cocycle condition on this set.

3.10. Assertion. The hypotheses and the construction guarantee that these values lie in the complement of $-I$.

3.11. This assertion will be proved below. Then in parallel with (2.10.5) we can define, for each $x \in \partial^{1} c_{\alpha}^{\sigma}$ and for each $s, 0 \leqq s \leqq 1$,

$$
v_{\alpha}^{\sigma}((1-s) \alpha+s x)=\mathfrak{g}(s),
$$

where $g$ is the unique shortest geodesic from $I$ to $v_{\alpha}^{\sigma}(x)$. The coherence of the procedure is proved by a transposition to this context of Lemmas 2.12-2.14. 
3.12. Proof of Assertion 3.10. Let us consider the worst case, when $\sigma$ is a 4-simplex, say $\sigma=\langle\alpha \beta \gamma \delta \varepsilon\rangle$, ordered as listed. Since $\alpha$ is lowest-ordered, the rules above give $v_{\alpha}^{\sigma}=I$ on $c_{\alpha}^{\sigma}$. For vertex $\beta, \varrho=\langle\alpha \beta\rangle$, and on $\partial^{1} c_{\beta}^{o}$ we have $v_{\beta}^{\sigma}=v_{\beta \alpha}^{*} v_{\alpha}^{\sigma} v_{\alpha^{\prime} \beta^{\prime}}^{*}$. By hypothesis $v_{\beta \alpha}^{*}=v_{\beta \alpha}$ is within $\pi / 8$ of $u_{\beta \alpha}$, and $v_{\alpha^{\prime} \beta^{\prime}}^{*}=v_{\alpha \beta}^{\prime}$ is within $\pi / 8$ of $u_{\alpha \beta}$. So on $\partial^{1} c_{\beta}^{o}$ the $v_{\beta}^{\sigma}$ values lie in $B(\pi / 4)$, the open ball of radius $\pi / 4$ about $I$. Since the extension is performed by coning from $I$, the new values also lie in $B(\pi / 4)$.

For vertex $\gamma, \varrho=\langle\alpha \beta \gamma\rangle$, and $\partial^{1} c_{\gamma}^{\varrho}=\left(c_{\gamma} \cap c_{\alpha}\right) \cup\left(c_{\gamma} \cap c_{\beta}\right)$. On the first set, $v_{\gamma}^{\sigma}$ $=v_{\gamma \alpha} v_{\alpha}^{\sigma} v_{\alpha \gamma}^{\prime}$. These values lie in $B(\pi / 4)$ just as was shown above. On the second, $v_{\gamma}^{\sigma}$ $=v_{\gamma \beta} v_{\beta}^{\sigma} v_{\beta \gamma}^{\prime}$. The first factor is within $\pi / 8$ of $u_{\gamma \beta}$; the second within $\pi / 4$ of $I$; and the third within $\pi / 8$ of $u_{\beta \gamma}$. So these values lie within $B(\pi / 2)$, and so do those of their extension to all of $c_{\gamma}^{\sigma}$.

Proceeding in the same way for vertices $\delta$ and $\varepsilon$, we find that the construction gives $v_{\delta}^{\sigma}$ mapping $c_{\delta}^{\sigma}$ into $B(3 \pi / 4)$ and that for $v_{\varepsilon}^{\sigma}($ here $\varrho=\sigma)$ the values on $\partial^{1} c_{\varepsilon}^{\sigma}$ all lie in $B(\pi)$. This completes the proof of the assertion and of Proposition 3.4.

3.13. Proof of Proposition 3.6. It is sufficient to prove the proposition under the extra hypothesis that $\mathbf{o}^{\prime}$ differs from $\mathbf{0}$ merely in the transposition of two $\mathbf{0}$-consecutive vertices, say $\gamma$ and $\delta$, such that $\gamma$ o-precedes $\delta$.

As in the proof of Proposition 3.4 we denote the vertices of $\Lambda \times[0,1]$ by $\alpha=(\alpha, 0)$ and $\alpha^{\prime}=(\alpha, 1)$. Here, however, we will work on a simplicial subdivision $\Lambda^{*}$ of $\Lambda \times[0,1]$. This subdivision, which is part of the "prism construction" in simplicial homology theory $[11,28]$, does not introduce any new vertices, and is defined as follows. Let $\sigma=\langle\alpha \beta \ldots \zeta\rangle$ be any simplex of $\Lambda$, its vertices written in increasing $\mathbf{o}$-order. Then $\sigma \times[0,1]$ is subdivided in $\Lambda^{*}$ to

$$
\left\{\left\langle\alpha \ldots \lambda \lambda^{\prime} \ldots \zeta^{\prime}\right\rangle \mid \lambda=\alpha, \ldots, \zeta\right\} \text {. }
$$

The new 1-simplexes of $\Lambda^{*}$ are (see Fig. 3.2)

$$
\left\{\left\langle\alpha \beta^{\prime}\right\rangle \mid\langle\alpha \beta\rangle \in \Lambda \text { and } \alpha \text { o-precedes } \beta\right\} \text {. }
$$

3.14. Next we define a local ordering $\mathrm{o}^{*}$ of the vertices of $\Lambda^{*}$ by requiring that $\mathrm{o}^{*}$ restrict to $\mathbf{o}$ on the vertices of $\Lambda \times\{0\}$ and to $\mathbf{o}^{\prime}$ on those of $\Lambda \times\{1\}$; and that all the vertices of $\Lambda \times\{0\}$ precede any vertex of $\Lambda \times\{1\}$. For example there are four possibilities for the $\mathbf{0}^{*}$-ordering of the vertices of $\tau^{*}=\left\langle\alpha \ldots \lambda \lambda^{\prime} \ldots \zeta^{\prime}\right\rangle$.

(1) If $\gamma$ and $\delta$ are both vertices of $\sigma$, then:

(a) if $\gamma$ o-precedes $\lambda$, then the $\mathbf{0}^{*}$-order is $\alpha \ldots \gamma \delta \ldots \lambda \lambda^{\prime} \ldots \zeta^{\prime}$;

(b) if $\gamma \mathbf{0}$-follows $\lambda$, then the $\mathbf{0}^{*}$-order is $\alpha \ldots \lambda \lambda^{\prime} \ldots \delta^{\prime} \gamma^{\prime} \ldots \zeta^{\prime}$;

(c) if $\gamma=\lambda$, then the $\mathbf{o}^{*}$-order is $\alpha \ldots \gamma \delta^{\prime} \gamma^{\prime} \ldots \zeta^{\prime}$.

(2) If $\gamma$ and $\delta$ are not both vertices of $\sigma$, then the $\mathbf{o}^{*}$-order is $\alpha \ldots \lambda \lambda^{\prime} \ldots \zeta^{\prime}$.

We observe that in case (a), $\lambda$ and $\lambda^{\prime}$ are consecutive, and otherwise the $\mathbf{0}^{*}$-ordering is the same as the o-ordering of the vertices of $\sigma$. Similarly in case (b), except that the $\mathbf{0}^{*}$-ordering is based on the $\mathbf{0}^{\prime}$-ordering of the vertices of $\sigma$. In case (2), these two descriptions coincide. Case (c) is exceptional in that $\gamma$ and $\gamma^{\prime}$ are not consecutive.

We now define an $\mathrm{SU}_{2}$-valued lattice gauge field $\mathbf{u}^{*}$ on $\Lambda^{*}$ by the rules: $u_{\alpha \alpha^{\prime}}^{*}=I$ for every vertex $\alpha$ of $\Lambda$; and $u_{\alpha \beta}^{*}=u_{\alpha \beta^{\prime}}^{*}=u_{\alpha^{\prime} \beta^{\prime}}^{*}=u_{\alpha \beta}$ for every 1-simplex $\langle\alpha \beta\rangle$ of $\Lambda$, ordered so that $\alpha \mathbf{0}$-precedes $\beta$. 
(a)
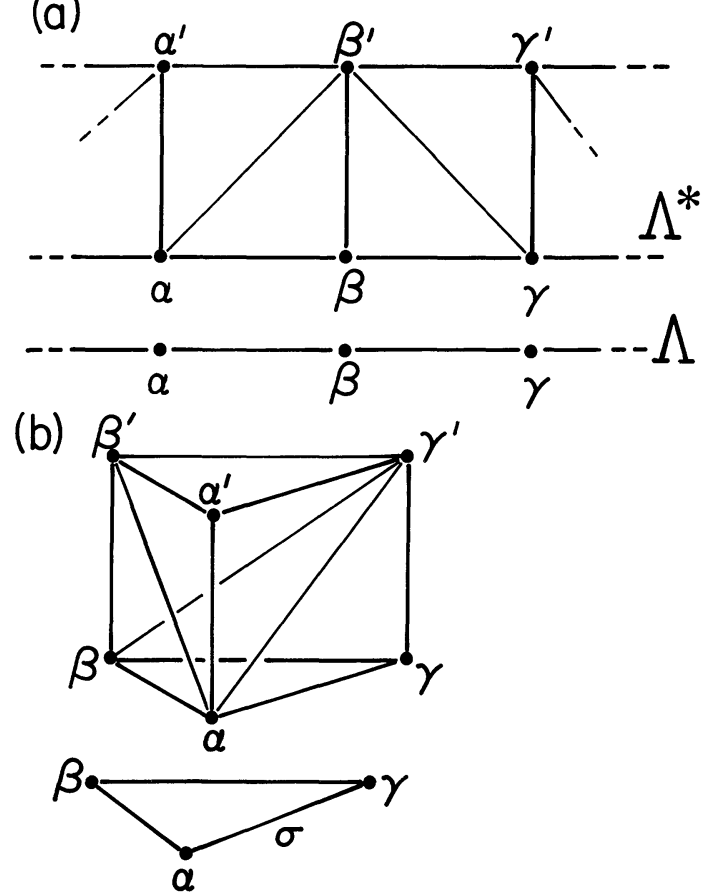

Fig. 3.2. a The subdivision $\Lambda^{*}$ of $\Lambda \times[0,1]$, where $\operatorname{dim} \Lambda=1$, with respect to a local ordering $\mathbf{o}$ in which $\alpha$ and $\gamma$ precede $\beta$. b A 2-simplex $\sigma$ of $\Lambda$ and the subdivision of $\sigma \times[0,1]$ in $\Lambda^{*}$; here $\alpha$ precedes $\beta$ and $\beta$ precedes $\gamma$ in $\mathbf{o}$

3.15. In order to apply the algorithm of Theorem 2.8 we first show that the 5-dimensional analogue of $B(\mathbf{o})$ holds for every simplex of $\Lambda^{*}$, namely:

$B\left(\mathbf{o}^{*}\right)$. For every simplex $\tau^{*}$ of dimension $\geqq 2$ in $\Lambda^{*}$,

$$
d\left(I, u_{\mathbf{o}^{*}}\left(\tau^{*}\right)\right)<\pi / 2 .
$$

We shall examine only the case that $\tau^{*}$ has the form $\left\langle\alpha \ldots \lambda \lambda^{\prime} \ldots \zeta^{\prime}\right\rangle$ discussed above; other cases, such as $\left\langle\alpha \ldots \lambda \mu^{\prime} \ldots \zeta^{\prime}\right\rangle$, are similar. In case (1a), and also in case (2),

$$
\begin{aligned}
u_{\mathbf{o}^{*}}^{*}\left(\tau^{*}\right) & =u_{\alpha \beta \ldots \kappa \lambda \lambda^{\prime} \mu^{\prime} \ldots \theta^{\prime} \zeta^{\prime} \alpha}^{*} \\
& =u_{\alpha \beta}^{*} \ldots u_{\kappa \lambda}^{*} u_{\lambda \lambda^{\prime}}^{*} u_{\lambda^{\prime} \mu^{\prime}}^{*} \ldots u_{\theta^{\prime} \zeta^{\prime}}^{*} u_{\zeta^{\prime} \alpha}^{*} \\
& =u_{\alpha \beta} \ldots u_{\kappa \lambda} I u_{\lambda \mu} \ldots u_{\theta \zeta} u_{\zeta \alpha} \\
& =u_{\alpha \beta \ldots \kappa \lambda \mu \ldots \theta \zeta \alpha} \\
& =u_{\mathbf{o}}(\sigma), \quad \text { where } \sigma \text { is a simplex of } \Lambda .
\end{aligned}
$$

Since $u$ is in $A_{2}$, the condition $B(\mathbf{o})$ holds for $\sigma$; and so $B\left(\mathbf{o}^{*}\right)$ holds for $\tau^{*}$ in this case. Case (1b) is similar: we obtain $u_{\mathbf{o}^{*}}^{*}\left(\tau^{*}\right)=u_{\mathbf{o}}(\sigma)$, and since $B\left(\mathbf{o}^{\prime}\right)$ holds for $\sigma, B\left(\mathbf{o}^{*}\right)$ 
follows for $\tau^{*}$. Lastly, in case (1c),

$$
\begin{aligned}
u_{\mathbf{o}^{*}}^{*}\left(\tau^{*}\right) & =u_{\alpha \beta \ldots \kappa \gamma \delta^{\prime} \gamma^{\prime} \mu^{\prime} \ldots \theta^{\prime} \zeta^{\prime} \alpha}^{*} \\
& =u_{\alpha \beta} \ldots u_{\kappa \gamma} u_{\gamma \delta} u_{\delta \gamma} u_{\gamma \mu} \ldots u_{\theta \zeta} u_{\zeta \alpha} \\
& =u_{\mathbf{o}}(\varrho), \quad \text { where } \varrho \text { is the face of } \sigma \text { opposite } \delta ;
\end{aligned}
$$

and $B\left(\mathbf{o}^{*}\right)$ follows in this case too.

3.16. We can now apply the algorithm used in the proof of Theorem 2.8; the increased dimension here does not affect the argument. We obtain a coordinate bundle $\mathbf{v}^{*}$ on $\Lambda^{*}$, which restricts to $\mathbf{v}$ on $\Lambda \times\{0\}$ and to $\mathbf{v}^{\prime}$ on $\Lambda \times\{1\}$ because $\mathbf{v}$ and $\mathbf{v}^{\prime}$ were constructed by the same algorithm applied to restrictions of the data $\Lambda^{*}, \mathbf{u}^{*}$ and $0^{*}$. As in the proof of Proposition 3.4, this implies that $\xi$ and $\xi^{\prime}$ are isomorphic, as required.

\section{Extensions and Improvements}

3.17. To Different Gauge Groups. Let $G$ be an arbitrary Lie group, and $r$ its radius of convexity (i.e. the largest number such that the open ball of radius $r$ about the identity is strictly convex). Then Theorem 2.5 holds for $G$-valued lattice gauge fields with any $\Delta \leqq r$; and Theorem 2.8 holds under a new hypothesis on $\mathbf{u}$ : namely that, on each 4-simplex, u satisfies the condition $B(\mathbf{o})$ with $\pi / 2$ replaced by $r$. Proposition 3.4 holds for $G$-valued lattice gauge fields and coordinate bundles satisfying (3.4.1) with $\pi / 8$ replaced by $r / 4$; and Proposition 3.6 holds if $A_{2}$ is defined as in (3.3.1) but with $\pi / 2$ replaced by $r$.

3.18. To Higher-Dimensional Simplicial Complexes. Let $\Lambda$ be any finitedimensional simplicial complex. Continuing with an arbitrary Lie group $G$ as above, Theorem 2.5 holds without further change; Theorem 2.8 should be further modified by requiring (2.7.1) for every simplex $\tau$ of dimension $\geqq 2$. In Proposition 3.4 the hypothesis on $\mathbf{v}$ is now (3.4.1) with $\pi / 8$ replaced by $r / \operatorname{dim} \Lambda$; no further change is necessary in Proposition 3.6.

3.19. In case $G=\mathrm{SU}_{2}$ hypotheses involving condition $B(\mathbf{o})$ can be weakened by replacing $\pi / 2$ with $\pi / 2+\varepsilon$, where $\varepsilon$ decreases with $\operatorname{dim} \Lambda$. For Theorem 2.8 $(\operatorname{dim} \Lambda=4)$ we calculate that we can use approximately $\pi / 2+0.3$ radians, and for Proposition $3.6, \pi / 2+0.2$ radians (in the definition of $A_{2}$ ). This allows us to construct examples having the standard unit quaternions $\mathbf{i}, \mathbf{j}, \mathbf{k}$ as plaquette products, knowing that the bundle produced by our algorithm will not depend on the choice of local ordering. On the other hand, the bound in the definition of $A_{2}$ cannot be allowed to exceed $2 \pi / 3$, as the following example shows.

3.20. Example. Here the lattice is $\partial \Delta^{5}$, the complex of proper faces of the 5-simplex. Topologically, this is a 4-sphere. Let us label the vertices $\alpha, \beta, \gamma, \delta, \varepsilon, \zeta$, and consider the $\mathrm{SU}_{2}$-valued lattice gauge field $\mathbf{u}$ on $\partial \Delta^{5}$ (see Fig. 3.3) defined by $u_{\alpha \beta}=\mathbf{j}^{\prime}, u_{\alpha \gamma}=\mathbf{k}^{\prime}$, $u_{\alpha \delta}=\mathbf{i}^{\prime}, u_{\alpha \varepsilon}=\boldsymbol{\omega}^{\prime}, u_{\beta \varepsilon}=-\mathbf{j}^{\prime \prime}$ and all other transporters $=\mathbf{1}$. Here $\mathbf{1}, \mathbf{i}, \mathbf{j}, \mathbf{k}$ refer to the standard unit quaternions, $\boldsymbol{\omega}=-1 / 2(\mathbf{1}+\mathbf{i}+\mathbf{j}+\mathbf{k})$, and the ' and "indicate small perturbations of the quaternion values, chosen so as to make $\mathbf{u}$ generic. The products $u_{a b c a}, u_{a b c d a}$, and $u_{a b c d e a}$, for $a, b, c, d, e$ in a 4-simplex, all lie within $2 \pi / 3$ (neglecting perturbations) of $\mathbf{1}$. 


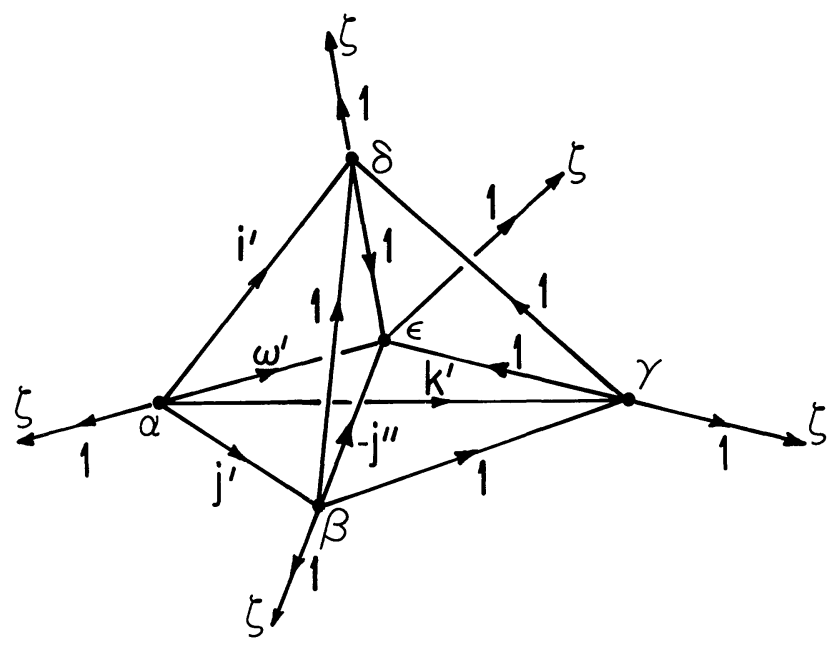

Fig. 3.3. $\mathrm{An} \mathrm{SU}_{2}$-valued lattice gauge field on $\partial \Delta^{5}$. (In this rendering of the 1-skeleton of $\partial \Delta^{5}$ the vertex $\zeta$ has been projected to infinity)

When our algorithm is applied to $\mathbf{u}$ and to the ordering $\alpha<\beta<\gamma<\delta<\varepsilon<\zeta$ it produces a nontrivial bundle $(Q=1)$, whereas the ordering $\beta<\alpha<\gamma<\delta<\varepsilon<\zeta$ gives a bundle with $Q=0$. The topological charge is calculated following the procedure described in the next section.

\section{The Calculation of Topological Charge}

4.1. If $\mathbf{u}$ is a $\mathrm{SU}_{2}$-valued lattice gauge field defined on a 4-dimensional simplicial complex $\Lambda$ and satisfying the continuity condition (2.7) with respect to some local ordering $\mathrm{o}$ of the vertices of $\Lambda$, then the algorithm of Theorem 2.8 produces an $\mathrm{SU}_{2}$ coordinate bundle $\mathbf{v}$ from $\mathbf{u}$ and $\mathbf{o}$. In this section we will show how this algorithm can be extended to yield the second Chern number of the principal bundle $\xi$ underlying v. In the range where $\xi$ is independent of the construction (e.g. in the set $A_{1}$ of (3.2)) this can be called the topological charge of $\mathbf{u}$.

For a principal $\mathrm{SU}_{2}$-bundle over a 4-complex, the second Chern number $C_{2}(\xi)$ coincides [19] with the Euler number of $\xi$, i.e. the obstruction to the existence of a section in $\xi$; in this context, obstruction has the following precise meaning.

4.2. Let $\pi: E(\xi) \rightarrow \Lambda$ be the projection from the total space of $\xi$ onto its base. A section over a subset $X \subset \Lambda$ is a continuous map $S: X \rightarrow E(\xi)$ such that $\pi(S(x))=x$ for every $x$ in $X$.

We will show how to construct a section $S$ over the 3 -skeleton $\Lambda_{(3)}$ of $\Lambda$. Choosing a trivialization $\Phi: \pi^{-1} \sigma \rightarrow \sigma \times \mathrm{SU}_{2}$ over a 4-simplex $\sigma$ of $\Lambda$ identifies $S \mid \partial \sigma$ with a map $S_{\sigma}: \partial \sigma \rightarrow \mathrm{SU}_{2}$, and it is clear that $S$ can be extended as a section over $\sigma$ if and only if $S_{\sigma}$, which is topologically a map between two 3-spheres, is nullhomotopic. What is less obvious is the following theorem.

Let us assume the $\sigma$ 's and the $\Phi$ 's have all been coherently oriented (orientations will be discussed more in detail below). 


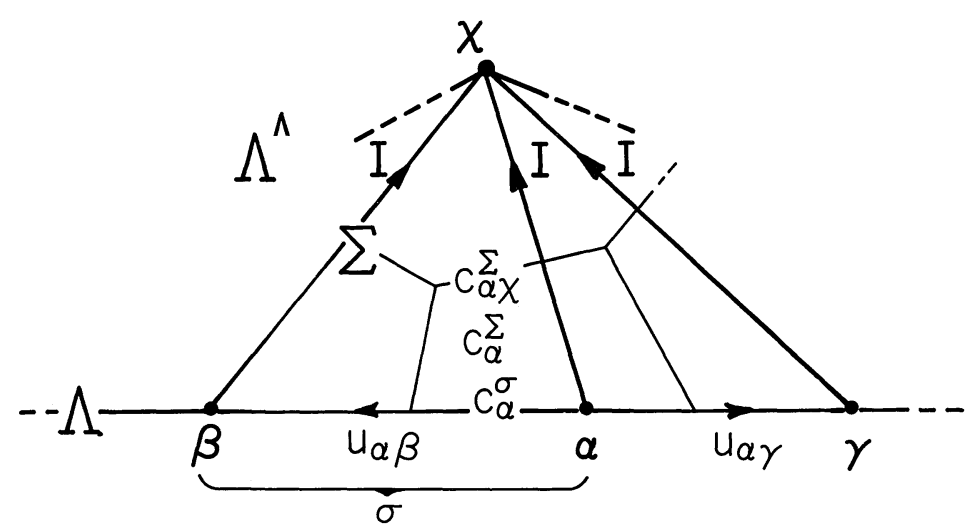

Fig. 4.1. The complex $\hat{\Lambda}$, its dual cells and the lattice gauge field $U$. Here $\Lambda$ is 1-dimensional. $\hat{\Lambda}$ is the cone from $\chi$ on $\Lambda$

4.3. Theorem $[19,27]$. Let $N_{\sigma} \in \pi_{3} \mathrm{SU}_{2}=\mathbf{Z}$ be the degree of $S_{\sigma}$. Then the integer $\sum_{\sigma \in A} N_{\sigma}$ does not depend on $S$ and is in fact the Euler number of $\xi$.

This explains how in our context $C_{2}(\xi)$ can be characterized as the obstruction to the existence of a section in $\xi$.

4.4. We will show presently that $C_{2}(\xi)$ can also be described as the obstruction to extending $\mathbf{v}$ over the augmented complex $\hat{\Lambda}$ formed by coning $\Lambda$ from a point $\chi$. (This complex has one new vertex $\chi$, one new 1-simplex $\langle\alpha \chi\rangle$ for each vertex $\alpha$ of $\Lambda$, etc.; see Fig. 4.1.) This last obstruction can be realized by the following construction. First extend $\mathbf{u}$ to a lattice gauge field $\mathbf{U}$ on $\hat{\Lambda}$ by defining $U_{\alpha \chi}=I$ for each new 1-simplex, and extend $\mathbf{o}$ to a local ordering $\mathbf{O}$ of the vertices of $\hat{\Lambda}$ by placing $\chi$ after all the vertices of $\Lambda$. In particular this defines a lattice gauge field and a local vertex ordering on the 4-skeleton $\hat{\Lambda}_{(4)}$. If $\mathbf{U}$ does not belong to the exceptional set $K(\mathbf{O})$ on $\hat{\Lambda}_{(4)}$ (this means additional measure-zero continuity-type conditions on $\mathbf{u}$; see below) then the algorithm of Theorem 2.8 may be applied to $\mathbf{U}$ and $\mathbf{O}$ to give an $\mathrm{SU}_{2}$-coordinate bundle $\mathbf{V}$ defined on $\hat{\Lambda}_{(4)}$.

4.5. Here is how to interpret $\mathbf{V}$ as a section $S$ over $\Lambda_{(3)}$. Let $\sigma$ be a $k$-simplex of $\Lambda_{(3)}$, so $k \leqq 3$, and $\Sigma=\chi * \sigma$ the corresponding $(k+1)$-simplex of $\hat{\Lambda}_{(4)}$. For any vertex $\alpha$ of $\sigma$, the set $c_{\alpha}^{\sigma}=\sigma \cap c_{\alpha}$ has modified barycentric coordinates $s_{\mu}, \ldots, s_{\lambda}$, where $\mu, \ldots, \lambda \neq \alpha$ are the other $k$ vertices of $\sigma$. The set $c_{\alpha \chi}^{\Sigma}=\Sigma \cap c_{\alpha} \cap c_{\chi}$ has modified barycentric coordinates with exactly the same names. Define $S_{\alpha}^{\sigma}: c_{\alpha}^{\sigma} \rightarrow \mathrm{SU}_{2}$ by $S_{\alpha}^{\sigma}\left(s_{\mu}, \ldots, s_{\lambda}\right)=V_{\alpha \chi}^{\Sigma}\left(s_{\mu}, \ldots, s_{\lambda}\right)$. We will write simply $S_{\alpha}^{\sigma}=V_{\alpha \chi}^{\Sigma}$.

4.6. Proposition. The maps $S_{\alpha}^{\sigma}$ fit together to give a section in $\xi$ over $\Lambda_{(3)}$.

Proof. First note that if $\alpha \in \sigma \cap \tau$, then on $c_{\alpha}^{\sigma} \cap c_{\alpha}^{\tau}$

$$
S_{\alpha}^{\sigma}=V_{\alpha \chi}^{\Sigma}=V_{\alpha \chi}^{\top}=S_{\alpha}^{\tau}, \text { where } \mathrm{T}=\chi * \tau,
$$

the middle equality from (2.10.6). 
Now suppose $x \in \sigma \cap c_{\alpha} \cap c_{\beta}$. Then

$$
\begin{aligned}
S_{\alpha}^{\sigma}(x) & =V_{\alpha \chi}^{\Sigma}(x) \\
& =\left(V_{\alpha \beta}^{\Sigma}(x) V_{\beta \alpha}^{\Sigma}(x)\right) \text { by }(2.10 .8) \\
& =v_{\alpha \beta}^{\sigma}(x) V_{\beta \chi}^{\Sigma}(x) \text { since } \mathbf{V} \text { extends } \mathbf{v} \\
& =v_{\alpha \beta}^{\sigma}(x) S_{\beta}^{\sigma}(x),
\end{aligned}
$$

i.e the $S_{\alpha}^{\sigma}$ s transform as the local coordinates of a section in $\xi$.

4.7. Note. The continuity hypothesis guarantees in fact that the algorithm will produce $V_{\alpha \chi}^{\Sigma}$ on any set of the form $c_{\alpha \chi}^{\Sigma}$ as long as $\operatorname{dim} \Sigma \leqq 4$ or $\operatorname{dim} \Sigma=5$ and $\alpha$ is not the first vertex of $\Sigma$. It follows that the section $S$ is defined over any set $c_{\alpha}^{\sigma}$ except when $\sigma=\langle 01234\rangle$ is 4-dimensional and $\alpha=0$. So the integer $N_{\sigma}$ of Theorem 4.3 becomes the homotopy class of $S \mid \partial c_{0}^{\sigma}$ or, equivalently, the homotopy class of $V_{0 \chi}^{\Sigma} \mid \partial c_{0 \chi}^{\Sigma} \Lambda$, where $\Sigma=\chi * \sigma=\langle 01234 \chi\rangle$.

4.8. Calculation of $N_{\sigma}$ (Beginning). The map $V_{0 \chi}^{\Sigma} \mid \partial c_{0 \chi}^{\Sigma}$ has a specific geometric form due to the algorithm. To write it explicitly, let us review the definitions made in the extension of $\mathbf{v}$ to $\hat{\Lambda}_{(4)}$.

$$
\begin{aligned}
& V_{4 \chi}^{\Sigma}=U_{4 \chi}=I, \\
& V_{3 \chi}^{\Sigma}=\mathfrak{g}_{34 \chi}\left(s_{4}\right) .
\end{aligned}
$$

(Note that $g_{34 x}$ is the unique shortest geodesic from $I$ to $u_{34}$.)

$$
\begin{gathered}
V_{2 \chi}^{\Sigma}=\mathfrak{h}_{234 \chi}\left(s_{3}, s_{4}\right), \\
V_{1 \chi}^{\Sigma}=\mathfrak{l}_{1234 \chi}\left(s_{2}, s_{3}, s_{4}\right) .
\end{gathered}
$$

Finally, on the boundary of $c_{0 \chi}^{\Sigma}, V_{0 \chi}^{\Sigma}$ is prescribed: on $\partial^{0} c_{0_{\chi}}^{\Sigma}$ by

$$
\begin{aligned}
& V_{0 \chi}^{\Sigma}\left(s_{4}=0\right)=\mathfrak{F}_{0123 \chi}\left(s_{1}, s_{2}, s_{3}\right), \\
& V_{0 \chi}^{\Sigma}\left(s_{3}=0\right)=\mathfrak{f}_{0124 \chi}\left(s_{1}, s_{2}, s_{4}\right), \\
& V_{0 \chi}^{\Sigma}\left(s_{2}=0\right)=\mathfrak{f}_{0134 \chi}\left(s_{1}, s_{3}, s_{4}\right), \\
& V_{0 \chi}^{\Sigma}\left(s_{1}=0\right)=\mathfrak{f}_{0234 \chi}\left(s_{2}, s_{3}, s_{4}\right) ;
\end{aligned}
$$

on $\partial^{1} c_{0, \mathcal{L}}^{\Sigma}$ by

$$
\begin{aligned}
& V_{0 \chi}^{\Sigma}\left(s_{4}=1\right)=V_{04}^{\Sigma} V_{4 \chi}^{\Sigma}=\mathfrak{f}_{01234}\left(s_{1}, s_{2}, s_{3}\right), \\
& V_{0 \chi}^{\Sigma}\left(s_{3}=1\right)=V_{03}^{\Sigma} V_{3 \chi}^{\Sigma}=\mathfrak{h}_{0123}\left(s_{1}, s_{2}\right) \mathfrak{g}_{34 \chi}\left(s_{4}\right), \\
& V_{0 \chi}^{\Sigma}\left(s_{2}=1\right)=V_{02}^{\Sigma} V_{2 \chi}^{\Sigma}=\mathfrak{g}_{012}\left(s_{1}\right) \mathfrak{h}_{234 \chi}\left(s_{3}, s_{4}\right), \\
& V_{0 \chi}^{\Sigma}\left(s_{1}=1\right)=V_{01}^{\Sigma} V_{1 \chi}^{\Sigma}=u_{01} \mathfrak{f}_{1234 \chi}\left(s_{2}, s_{3}, s_{4}\right) .
\end{aligned}
$$

Note that implicit in these definitions is a partition of each face of $\partial c_{0 \chi}^{\Sigma}$ into subpolyhedra. When the map is of type $\mathfrak{f}$ or $u \cdot \mathfrak{l}$, these are four simplexes and a square- 
based pyramid (see Fig. 2.5); when it is of type $\mathfrak{h} \cdot \mathfrak{g}$ or $\mathfrak{g} \cdot \mathfrak{h}$, these are two triangular prisms (compare with Fig. 2.4).

4.9. The existence of all the unique shortest geodesics required for the definition of these maps requires an additional set of continuity conditions. In terms of $\mathbf{u}$, on each 3 -simplex $\langle 0123\rangle$ of $\Lambda$ one of the two following conditions, coming from applying $A(\mathbf{O})$ and $B(\mathbf{O})$ to $\langle 0123 \chi\rangle$, must hold.

* from $A(\mathbf{o})$. The following five sets of elements must be linearly independent:

$$
\begin{array}{cccc}
I, & u_{0120}, & u_{012}, & u_{0123}, \\
I, & u_{01}, & u_{012}, & u_{0123}, \\
I, & u_{01}, & u_{013}, & u_{0123}, \\
I, & u_{03}, & u_{013}, & u_{0123}, \\
I, & u_{03}, & u_{023}, & u_{0123}
\end{array}
$$

* from $B(\mathbf{O})$. The following elements must all lie within $\pi / 2$ of $I$ :

$$
\begin{aligned}
& u_{01}, \quad u_{02}, \quad u_{03}, u_{12}, u_{13}, u_{23} \text {, } \\
& u_{012}, u_{013}, u_{023}, u_{123}, u_{0123} \text {. }
\end{aligned}
$$

4.10. Note. Condition $A(\mathbf{O})$ is satisfied on the complement of a set of measure zero in the space of all lattice gauge fields. Note however that the new conditions are not gauge-invariant; also, $B(\mathbf{O})$ is a much stronger condition than $B(\mathbf{O})$. Clearly $B(\mathbf{O})$ always holds in the "continuum limit;" but if $\mathbf{u}$ satisfies $B(\mathbf{O})$ for every 4-simplex of $\hat{\Lambda}$, then it can be shown that $C_{2}(\mathbf{u})=0$ (compare with [23, Proposition 1.12]).

4.11. Orientations. Nobody likes to think about orientations. Here it is unavoidable, because the $V_{0 \chi}^{\Sigma}$ 's have an intrinsic orientation coming from the local ordering, and this must be compared with a global orientation of $\Lambda$ and of $\xi$ if we want the various $N_{\sigma}$ 's to add up correctly.

Suppose $\Lambda$ is an oriented 4-dimensional simplicial manifold. One way of defining "oriented" is to begin with the concept of an orientation of a simplex: this is the choice of an equivalence class of vertex-orderings, where two are equivalent if they differ by an even permutation. An orientation of $\Lambda$ is then the choice of an orientation for each 4-simplex of $\Lambda$ (we will call the distinguished orientations "positive") in such a way that two adjacent simplexes induce opposite orientations on their common 3-face. To say that $\Lambda$ is oriented means that such a choice can be and has been made.

4.12. We can define an orientation of a smooth manifold $M$ as a continuous assignment of a sign ( + or - ) to each tangent $n$-frame. If $M$ is triangulated as a simplicial manifold $\Lambda$, then an orientation of $\Lambda$ gives one of $M$ : if a frame $v_{1}, \ldots, v_{n}$ is at a point of a simplex $\sigma=\langle 012 \ldots n\rangle$ with vertices thus positively ordered, slide it over to 0 and compare it with the frame $\overrightarrow{01}, \overrightarrow{02}, \ldots, \overrightarrow{0 n}$. It is easy to check that the sign so determined does not depend on $\sigma$.

4.13. Induced orientations: we follow the convention that the orientation induced on a boundary face is that which, preceded by an outward-pointing vector, gives the orientation of the interior. 
4.14. Next suppose $\xi$ is an oriented principal $\mathrm{SU}_{2}$-bundle over $\Lambda$, in the following sense. Take the group $\mathrm{SU}_{2}$ as being oriented as a smooth manifold, say with the orientation induced from the standard orientation of $\mathbf{R}^{4}$ (see 4.23). Then the local fiber coordinates may all be coherently oriented, since any two of them differ by multiplication by an element of $\mathrm{SU}_{2}$ (this preserves orientation). We suppose that a coherent orientation has been chosen, and we call those fiber coordinates "positively oriented."

4.15. Calculation of $N_{\sigma}$ (Continued). We now have an oriented $\mathrm{SU}_{2}$-bundle $\xi$ over an oriented 4-dimensional simplicial manifold $\Lambda$; suppose in addition we have a local ordering $\mathbf{o}$ of the vertices of $\Lambda$. Given a 4-simplex $\sigma=\langle 01234\rangle$ of $\Lambda$, the vertices listed in their o-ordering, let $\varepsilon_{\mathbf{o}}(\sigma)=+1$ if the frame $\left.\overrightarrow{(01}, \overrightarrow{02}, \overrightarrow{03}, \overrightarrow{04}\right)$ is positively oriented, and -1 otherwise. Let $c_{0}^{\sigma}$ be oriented by $(\overrightarrow{01}, \overrightarrow{02}, \overrightarrow{03}, \overrightarrow{04})$ and give $\partial c_{0 \chi}^{\Sigma}\left[\right.$ identified with $\partial c_{0}^{\sigma}$ as in (4.5)] the induced orientation. Let $N_{\mathbf{o}}(\sigma)$ be the degree of the map $V_{0 \chi}^{\Sigma}: \partial c_{0 \chi}^{\Sigma} \rightarrow \mathrm{SU}_{2}$ with respect to that orientation and a positive fiber coordinate. Then we may take

$$
N_{\sigma}=\varepsilon_{\mathbf{o}}(\sigma) N_{\mathbf{o}}(\sigma)
$$

The rest of this section will be devoted to the calculation of $N_{\mathbf{o}}(\sigma)$.

4.16. Our algorithm for computing the homotopy class of $V_{0 \chi}^{\Sigma}$ is based [12] on picking a point $y$ in $\mathrm{SU}_{2}$ which is generic with respect to the image of $V_{0 \chi}^{\Sigma}$ in a sense to be made precise soon. For now it is enough that $y$ be chosen so that $\left(V_{0 \chi}^{\Sigma}\right)^{-1}(y)$ is a finite set of points $x_{1}, \ldots, x_{n}$ and $V_{0 \chi}^{\Sigma}$ is a local homeomorphism at each $x_{v}$. Then we assign to each $x_{v}$ a number $\phi_{v}$, which is 1 or -1 according as $V_{0 \chi}^{\Sigma}$ preserves or reverses orientation at $x_{v}$. Finally, the value of $N_{\mathrm{o}}(\sigma)$ is $\phi_{1}+\ldots+\phi_{n}$.

To compute the numbers $\phi_{v}$ we exploit the precise geometry of the construction of $V_{0 \chi}^{\Sigma}$. As remarked in (4.8), this map implicitly subdivides $\partial c_{0}^{\sigma}$ into a complex $K$ whose 3-cells are simplexes, pyramids or prisms. On cells of each type, $V_{0 \chi}^{\Sigma}$ is geometrically the same; for example $V_{0 \chi}^{\Sigma}$ maps each simplex onto a convex, geodesic simplex in $\mathrm{SU}_{2}$.

4.17. The genericity requirement on $y$ can now be stated: in addition to the conditions given above, the $x_{v}$ must all lie in the interiors of the 3 -cells of $K$; that is, $y$ must be in general position with respect to the image under $V_{0 \chi}^{\Sigma}$ of the 2-skeleton of $K$.

\subsection{Our program consists of the following steps.}

(1) Using just the local ordering $\mathbf{0}$, we shall define an "intrinsic" orientation for each 3-cell $D$ of $K$.

(2) We then calculate the relative orientation $\varepsilon\left(D ; \partial c_{0}^{\sigma}\right)$ which is +1 or -1 according as the orientation of $D$ agrees with the orientation of $\partial c_{0}^{\sigma}$ described above or not.

Then, depending on whether $D$ is of type $I$ (simplex), type $I I$ (pyramid) or type III (prism) we shall give

(3) a criterion, satisfied on an open, dense set in $\mathrm{SU}_{2}$, for when a point $y$ is generic with respect to $V_{0 \chi}^{\Sigma} \mid D$. 
We shall also give and justify algorithms to determine

(4) the number $n(D)$ of points $x_{v}$ in $\left(V_{0 \chi}^{\Sigma}\right)^{-1}(y) \cap D$ (this number will turn out to be 0,1 or 2 );

(5) and when $n(D)=1$, the orientation $\varepsilon_{x(D)}$ of $V_{0 \chi}^{\Sigma}$ (considered as a map from $D$ to $\mathrm{SU}_{2}$ ) at the single $x_{v}=x(D)$; this is +1 if $V_{0 \chi}^{\Sigma}$ preserves orientation at $x(D)$, and -1 otherwise. [We will show that when $n(D)=2$, the map $V_{0 \chi}^{\Sigma}$ has opposite orientations at the two inverse image points.] Finally,

$$
N_{\mathbf{o}}(\sigma)=\sum_{D: n(\mathrm{D})=1} \varepsilon\left(D: \partial c_{0}^{\sigma}\right) \varepsilon_{x(\mathrm{D})}
$$

4.19. First some notation for the vertices of $c_{0}^{\sigma}$. In the modified barycentric coordinates $\left(s_{1}, s_{2}, s_{3}, s_{4}\right)$ of (2.9) these are the 16 points where each coordinate is 0 or 1 . Each vertex $v$ may then be identified by the subset $H \subset\{1,2,3,4\}$ made up of the indices of the coordinates which it has equal to 1 . It will be convenient to label $v$ by the set $H^{\prime}=\{0\} \cup H$, the elements written in increasing order, because then the image $V_{0 \chi}^{\Sigma}(v)$ can be read off directly from the label.

$$
V_{0 \chi}^{\Sigma}\left(v_{H^{\prime}}\right)=\left\{\begin{array}{lll}
I & \text { if } & H^{\prime}=\{0\} \\
u_{0 i j \ldots k} & \text { if } & H^{\prime}=\{0, i, j, \ldots, k\}, i<j<\ldots<k .
\end{array}\right.
$$

[For example, the vertex with $s_{1}=s_{2}=s_{4}=1, s_{3}=0$ would be labelled 0124 , and $\left.V_{0 \chi}^{\Sigma}(0124)=u_{0124}=u_{01} u_{12} u_{24} \cdot\right]$

4.20. We will label the 8 faces of $c_{0}^{\sigma}$ by $C^{i}=\left\{s_{i}=1\right\}$ and $C_{i}=\left\{s_{i}=0\right\}$. Each of these faces has its own intrinsic orientation determined by the local ordering: $C^{i}$ and $C_{i}$ have coordinates $s_{j}, s_{k}, s_{l}$ with $j<k<l$ in the ordering; we will orient them by the ordered basis $\left(\partial / \partial s_{j}, \partial / \partial s_{k}, \partial / \partial s_{l}\right)$.

4.21. Finally each 3-cell $D$ of $K$ has an intrinsic orientation; the simplest way to describe it is to say that it is determined by the first four of the vertices of $D$ as they are listed in the table below. If these vertices are $v_{0}, v_{1}, v_{2}, v_{3}$, in that order, then the orientation is given by either one of the equivalent 3 -frames $\left(\overrightarrow{v_{0} v_{1}}, \overrightarrow{v_{0} v_{2}}, \overrightarrow{v_{0} v_{3}}\right)$ or $\left(\vec{v}_{0} v_{1}, \vec{v}_{1} \vec{v}_{2}, \vec{v}_{2} \vec{v}_{3}\right)$

4.22. The following table gives for each 3-cube $C \subset \partial c_{0}^{\sigma}$ the corresponding $\left.V_{0_{\chi}}^{\Sigma}\right|_{C}$ and lists the 3-cells $D$ which it contains. Each cell is identified by its vertices; its combinatorial type is also noted. In addition the table lists for each $C$ the sign $\varepsilon\left(C, \partial c_{0}^{\sigma}\right)$ relating its intrinsic orientation to that of $\partial c_{0}^{\sigma}$, and for each $D$ the sign $\varepsilon(D, C)$ relating its intrinsic orientation to that of $C$. The $\operatorname{sign} \varepsilon\left(D, \partial c_{0}^{\sigma}\right)$ is the product of these two.

Before we can continue our program we need some more notation.

4.23. Notation for $\mathbf{R}^{4}$ and $S^{3}$. The rest of our program makes use of the geometry of $\mathrm{SU}_{2}$ which we identify with the group of unit quaternions, geometrically the sphere $S^{3}$ of radius 1 in $\mathbf{R}^{4}$.

The term line will mean an affine line in $\mathbf{R}^{4}$, with $L\left[y_{1}, y_{2}\right]$ the line through points $y_{1}$ and $y_{2}$. A segment is a closed interval on a line; $\left[y_{1}, y_{2}\right]$ means the 
Table 4.1. Combinatorial type and relative orientation of the 3-cells $D$ of $\partial c_{0}^{\sigma}$. Here $\sigma=\langle 01234\rangle$ is a simplex of $\Lambda$, its vertices so ordered. The algorithm splits $\partial c_{0}^{\sigma}$ into 8 cubical faces, the $C^{i}$ and $C_{i}$, and maps each of them into $\mathrm{SU}_{2}$ either as the union of a pyramid and four simplexes or as the union of two prisms. A vertex labelled 0 is mapped to $I$, a vertex labelled $a b$ to $u_{a b}$, a vertex labelled $a b c$ to $u_{a b c}=u_{a b} u_{b c}$, etc.

\begin{tabular}{|c|c|c|c|c|}
\hline 3-cube $C$ & $\varepsilon\left(C, \partial c_{0}^{\sigma}\right)$ & Type of $D$ & Vertices of $D$ & $\varepsilon(D, C)$ \\
\hline $\begin{array}{l}\mathrm{C}_{1} \\
\left(\mathfrak{f}_{0234 \chi}\right)\end{array}$ & -1 & $\begin{array}{l}\text { Pyramid } \\
\text { Simplex } \\
\text { Simplex } \\
\text { Simplex } \\
\text { Simplex }\end{array}$ & $\begin{array}{l}0,03,023,0234,034 \\
0,02,023,0234 \\
0,02,024,0234 \\
0,04,024,0234 \\
0,04,034,0234\end{array}$ & $\begin{array}{l}-1 \\
+1 \\
-1 \\
+1 \\
-1\end{array}$ \\
\hline $\begin{array}{l}C_{2} \\
\left(\mathfrak{f}_{0134 \chi}\right)\end{array}$ & +1 & $\begin{array}{l}\text { Pyramid } \\
\text { Simplex } \\
\text { Simplex } \\
\text { Simplex } \\
\text { Simplex }\end{array}$ & $\begin{array}{l}0,03,013,0134,034 \\
0,01,013,0134 \\
0,01,014,0134 \\
0,04,014,0134 \\
0,04,034,0134\end{array}$ & $\begin{array}{l}-1 \\
+1 \\
-1 \\
+1 \\
-1\end{array}$ \\
\hline $\begin{array}{l}C_{3} \\
\left(\mathfrak{f}_{0124 \chi}\right)\end{array}$ & -1 & $\begin{array}{l}\text { Pyramid } \\
\text { Simplex } \\
\text { Simplex } \\
\text { Simplex } \\
\text { Simplex }\end{array}$ & $\begin{array}{l}0,02,012,0124,024 \\
0,01,012,0124 \\
0,01,014,0124 \\
0,04,014,0124 \\
0,04,024,0124\end{array}$ & $\begin{array}{l}-1 \\
+1 \\
-1 \\
+1 \\
-1\end{array}$ \\
\hline $\begin{array}{l}C_{4} \\
\left(\mathfrak{f}_{0123 x}\right)\end{array}$ & +1 & $\begin{array}{l}\text { Pyramid } \\
\text { Simplex } \\
\text { Simplex } \\
\text { Simplex } \\
\text { Simplex }\end{array}$ & $\begin{array}{l}0,02,012,0123,023 \\
0,01,012,0123 \\
0,01,013,0123 \\
0,03,013,0123 \\
0,03,023,0123\end{array}$ & $\begin{array}{l}-1 \\
+1 \\
-1 \\
+1 \\
-1\end{array}$ \\
\hline $\begin{array}{l}C^{1} \\
\left(u_{01}^{\mathfrak{F}}{ }_{1234 \chi}\right)\end{array}$ & +1 & $\begin{array}{l}\text { Pyramid } \\
\text { Simplex } \\
\text { Simplex } \\
\text { Simplex } \\
\text { Simplex }\end{array}$ & $\begin{array}{l}01,013,0123,01234,0134 \\
01,012,0123,01234 \\
01,012,0124,01234 \\
01,014,0124,01234 \\
01,014,0134,01234\end{array}$ & $\begin{array}{l}-1 \\
+1 \\
-1 \\
+1 \\
-1\end{array}$ \\
\hline $\begin{array}{l}C^{2} \\
\left(\mathfrak{g}_{012} \mathfrak{h}_{234 \chi}\right)\end{array}$ & -1 & $\begin{array}{l}\text { Prism } \\
\text { Prism }\end{array}$ & $\begin{array}{l}02,012,0123,01234,023,0234 \\
02,012,0124,01234,024,0234\end{array}$ & $\begin{array}{l}+1 \\
-1\end{array}$ \\
\hline $\begin{array}{l}C^{3} \\
\left(\mathfrak{h}_{0123} \mathfrak{g}_{34 \chi}\right)\end{array}$ & +1 & $\begin{array}{l}\text { Prism } \\
\text { Prism }\end{array}$ & $\begin{array}{l}03,013,0123,01234,034,0134 \\
03,023,0123,01234,034,0234\end{array}$ & $\begin{array}{l}+1 \\
-1\end{array}$ \\
\hline $\begin{array}{l}C^{4} \\
\left(\mathfrak{f}_{01234}\right)\end{array}$ & -1 & $\begin{array}{l}\text { Pyramid } \\
\text { Simplex } \\
\text { Simplex } \\
\text { Simplex } \\
\text { Simplex }\end{array}$ & $\begin{array}{l}04,024,0124,01234,0234 \\
04,014,0124,01234 \\
04,014,0134,01234 \\
04,034,0134,01234 \\
04,034,0234,01234\end{array}$ & $\begin{array}{l}-1 \\
+1 \\
-1 \\
+1 \\
-1\end{array}$ \\
\hline
\end{tabular}

segment with endpoints $y_{1}$ and $y_{2}$. The term geodesic will stand for a closed, minimal geodesic in $S^{3}$; if $y_{1}, y_{2}$ are points on $S^{3}, y_{1} \neq-y_{2}$, then $s\left[y_{1}, y_{2}\right]$ will represent the unique minimal geodesic between them.

Let $\mathbf{0}$ denote the origin in $\mathbf{R}^{4}$. For any set $X \subset \mathbf{R}^{4}$, the notation $c X$ represents the infinite cone on $X: c X=\{t y \mid y \in X, 0 \leqq t<\infty\}$. 
We set $s(X)=c X \cap S^{3}$. This defines a map $s: \mathbf{R}^{4}-\{\mathbf{0}\} \rightarrow S^{3}$; note that $s(X)$ $=s(c X)$. For example, if $\left[y_{1}, y_{2}\right]$ is a segment which does not contain $\mathbf{0}$, with $y_{1}, y_{2}$ on $S^{3}$, then $s\left(\left[y_{1}, y_{2}\right]\right)$ is the geodesic $s\left[y_{1}, y_{2}\right]$.

More generally, suppose that $X$ is convex and does not contain $\mathbf{0}$. Then $s(X)$ is strictly convex; that is, any $y_{1}, y_{2}$ in $s(X)$ can be joined by a unique (minimal) geodesic (in $S^{3}$ ) which lies in $S(X)$.

Finally, if $y_{1}, y_{2}, y_{3}, y_{4}$ are points in $\mathbf{R}^{4}, \operatorname{det}\left(y_{1}, y_{2}, y_{3}, y_{4}\right)$ means the determinant of the matrix with those four vectors as columns; if that matrix is nonsingular, then $\operatorname{sdet}\left(y_{1}, y_{2}, y_{3}, y_{4}\right)=+1$ or -1 is its sign. For $a \in \mathbf{R}^{4}$, let $\operatorname{det}_{i}(\mathrm{a}$; $\left.y_{1}, y_{2}, y_{3}, y_{4}\right)$ be the determinant formed by replacing $y_{i}$ with $a$.

If $\operatorname{det}\left(y_{1}, y_{2}, y_{3}, y_{4}\right) \neq 0$, then $\left(y_{1}, y_{2}, y_{3}, y_{4}\right)$ is a basis for $\mathbf{R}^{4}$, and the coordinates of $a$ in this basis are

$$
t_{i}(a)=\operatorname{det}_{i}\left(a ; y_{1}, y_{2}, y_{3}, y_{4}\right) / \operatorname{det}\left(y_{1}, y_{2}, y_{3}, y_{4}\right) .
$$

4.24. Calculation of $N_{\sigma}$ (Continued). Given a generic $y \in S^{3}$, the calculation has been reduced, by (4.15.1), (4.18.1) and the orientation coefficients given in the table, to the computation of $n(D)$ and, where appropriate, $\varepsilon_{x(D)}$ for a 3-cell $D$ of the complex $K$.

We now continue with parts (3)-(5) of the program of (4.18) in the three cases: I ( $D$ is a simplex), II ( $D$ is a pyramid) and III ( $D$ is a prism). Suppose $D$ has vertices $v_{0}$, $v_{1}, \ldots$ listed in the order given in Table 4.1.

Notation. In what follows we will shorten " $V_{0 x}^{\Sigma}$ " to " $V$ ".

4.25. Case $I$ : $D$ is a simplex, with vertices $v_{0}, v_{1}, v_{2}, v_{3}$. Set $y_{i}=V\left(v_{i}\right)$. Then $V(D)$ is the convex hull in $S^{3}$ of $y_{0}, y_{1}, y_{2}, y_{3}$. The continuity hypothesis guarantees that $y_{0}$, $y_{1}, y_{2}, y_{3}$ are in general position, so $V(D)$ is a strictly convex spherical 3-simplex.

(I.3) A point $y \in S_{3}$ is generic with respect to $V(D)$ provided no $t_{i}(y)=0$, $i=0,1,2,3$, where $t_{i}$ is defined as in (4.23). This condition is satisfied on an open, dense set in $S^{3}$.

(I.4) $y \in V(D)$ if and only if $y \in c V(D)$, which happens if and only if all $t_{i}(y)$ $>0$; and then $n(D)=1$.

(I.5) In Case $I, V$ either preserves or reverses orientation simultaneously at all points of $D$, according as $\operatorname{det}\left(y_{0}, y_{1}, y_{2}, y_{3}\right)$ is positive or negative. Hence $\varepsilon_{x(D)}=\operatorname{sdet}\left(y_{0}, y_{1}, y_{2}, y_{3}\right)$.

4.26. Case II: $D$ is a pyramid with cone point $v_{0}$ and base the square $R$ with vertices $v_{1}, v_{2}, v_{3}, v_{4}$ (in cyclic order).

Again, set $y_{i}=V\left(v_{i}\right)$. The continuity hypothesis guarantees that $y_{1}, y_{2}, y_{3}, y_{4}$ are in general position in $\mathbf{R}^{4}$. Let $t_{1}, \ldots, t_{4}$ be coordinates with respect to this basis, as in (4.23).

The 3-cell $D$ (see Fig. 2.5) is part of a 3-cube $C$ parametrized by $\left(s_{\alpha}, s_{\beta}, s_{\gamma}\right)$; where, in the $H^{\prime}$-notation, $v_{1}=v_{0} \cup\{\alpha\}, v_{2}=v_{1} \cup\{\beta\}, v_{4}=v_{1} \cup\{\gamma\}$ (and $v_{3}=v_{1} \cup\{\beta, \gamma\}$ ). Here $s_{\alpha}$ is 0 at $v_{0}$ and 1 on $R$, and $D$ has the structure of a cone with base $R: D=\left\{\left(s_{\alpha}\right.\right.$, 
$\left.\left.s_{\beta}, s_{\gamma}\right) \mid 0 \leqq s_{\beta}, s_{\gamma} \leqq s_{\alpha} \leqq 1\right\}$. If $x=\left(s_{\alpha}, s_{\beta}, s_{\gamma}\right)$, and $x \neq v_{0}$, set $v=\left(1, s_{\beta} / s_{\alpha}, s_{\gamma} / s_{\alpha}\right) \in R$; then $x=\left(1-s_{\alpha}\right) v_{0}+s_{\alpha} v$. On $R, s_{\beta}$ and $s_{\gamma}$ run from 0 at $v_{1}$ to 1 at $v_{2}$ and $v_{4}$ respectively.

4.27. We shall need the equation of the map $V: R \rightarrow S^{3}$. Now $V$ was defined on $R$ as the product of two geodesics:

$$
V\left(1, s_{\beta}, s_{\gamma}\right)=\mathfrak{g}_{\beta}\left(s_{\beta}\right) \mathfrak{g}_{\gamma}\left(s_{\gamma}\right) .
$$

Since multiplication by a fixed unit quaternion is an isometry, as $s_{\gamma}$ varies the length of the geodesic $s_{\beta} \rightarrow \mathfrak{g}_{\beta}\left(s_{\beta}\right) \mathfrak{g}_{\gamma}\left(s_{\gamma}\right)$ is a constant, say $\theta_{\beta}$; and similarly the length of each $\mathfrak{g}_{\beta}\left(s_{\beta}\right) \cdot \mathfrak{g}_{\gamma}$ is a constant $\theta_{\gamma}$. Thus $\theta_{\beta}$ is the spherical distance, or angle, between $y_{1}$ and $y_{2}$, and also between $y_{4}$ and $y_{3}$; while $\theta_{\gamma}$ is the angle between $y_{1}$ and $y_{4}$, and also between $y_{2}$ and $y_{3}$. It now follows that

$$
\begin{aligned}
& V\left(1, s_{\beta}, 0\right)=\frac{\sin \left(1-s_{\beta}\right) \theta_{\beta}}{\sin \theta_{\beta}} y_{1}+\frac{\sin s_{\beta} \theta_{\beta}}{\sin \theta_{\beta}} y_{2}, \\
& V\left(1, s_{\beta}, 1\right)=\frac{\sin \left(1-s_{\beta}\right) \theta_{\beta}}{\sin \theta_{\beta}} y_{4}+\frac{\sin s_{\beta} \theta_{\beta}}{\sin \theta_{\beta}} y_{3} .
\end{aligned}
$$

Hence

$$
\begin{aligned}
V\left(1, s_{\beta}, s_{\gamma}\right) & =\frac{\sin \left(1-s_{\gamma}\right) \theta_{\gamma}}{\sin \theta_{\gamma}} V\left(1, s_{\beta}, 0\right)+\frac{\sin s_{\gamma} \theta_{\gamma}}{\sin \theta_{\beta}} V\left(1, s_{\beta}, 1\right) \\
& =\sum t_{i} y_{i}, \quad \text { where } \\
t_{1} & =\left[\sin \left(\left(1-s_{\beta}\right) \theta_{\beta}\right) \sin \left(\left(1-s_{\gamma}\right) \theta_{\gamma}\right)\right] / \sin \theta_{\beta} \sin \theta_{\gamma}, \\
t_{2} & =\left[\sin \left(s_{\beta} \theta_{\beta}\right) \sin \left(\left(1-s_{\gamma}\right) \theta_{\gamma}\right)\right] / \sin \theta_{\beta} \sin \theta_{\gamma}, \\
t_{3} & =\left[\sin \left(s_{\beta} \theta_{\beta}\right) \sin \left(s_{\gamma} \theta_{\gamma}\right)\right] / \sin \theta_{\beta} \sin \theta_{\gamma}, \\
t_{4} & =\left[\sin \left(\left(1-s_{\beta}\right) \theta_{\beta}\right) \sin \left(s_{\gamma} \theta_{\gamma}\right)\right] / \sin \theta_{\beta} \sin \theta_{\gamma} .
\end{aligned}
$$

4.28. Using the coordinates $\left(t_{1}, t_{2}, t_{3}, t_{4}\right)$ with respect to the basis $y_{1}, \ldots, y_{4}$, define $q: \mathbf{R}^{4} \rightarrow \mathbf{R}$ by

$$
q\left(t_{1}, t_{2}, t_{3}, t_{4}\right)=t_{1} t_{3}-t_{2} t_{4}
$$

Then $q \circ V=0$ on $R$, so $V(R)$ lies in the variety $\{q=0\}$, which is a cone $c Q$ since $q$ is a homogeneous polynomial. We may take $Q=s(c Q)$, so $V(R)$ is a portion of $Q$. In fact $V(R)=\left\{\left(t_{1}, t_{2}, t_{3}, t_{4}\right) \in S^{3} \mid q\left(t_{1}, t_{2}, t_{3}, t_{4}\right)=0\right.$ and all $\left.t_{i} \geqq 0\right\}$ (see Fig. 4.2.).

4.29. The continuity hypothesis guarantees that $\pm y_{0} \notin Q$; so for each $z$ in $Q$ there is a unique shortest geodesic from $y_{0}$ to $z$. Let $A(z)$ be the angle between $y_{0}$ and $z$. Then the extension of $V$ over $D$ is given by

$$
V(x)=\frac{\sin \left(\left(1-s_{\alpha}\right) A(z)\right)}{\sin A(z)} y_{0}+\frac{\sin \left(s_{\alpha} A(z)\right)}{\sin A(z)} V(v),
$$

where $x=\left(s_{\alpha}, s_{\beta}, s_{\gamma}\right), v=\left(1, s_{\beta} / s_{\alpha}, s_{\gamma} / s_{\alpha}\right)$ as above, and $z=V(v)$.

4.30. Let $z=\left(t_{1}, t_{2}, t_{3}, t_{4}\right)=\sum t_{i} y_{i}$. The differential $\left.\mathrm{d} q\right|_{z}$ has components $\left(t_{3},-t_{4}\right.$, $\left.t_{1},-t_{2}\right)$ in the basis of the cotangent space at $z$ dual to the basis $\left(y_{1}, y_{2}, y_{3}, y_{4}\right)$. 


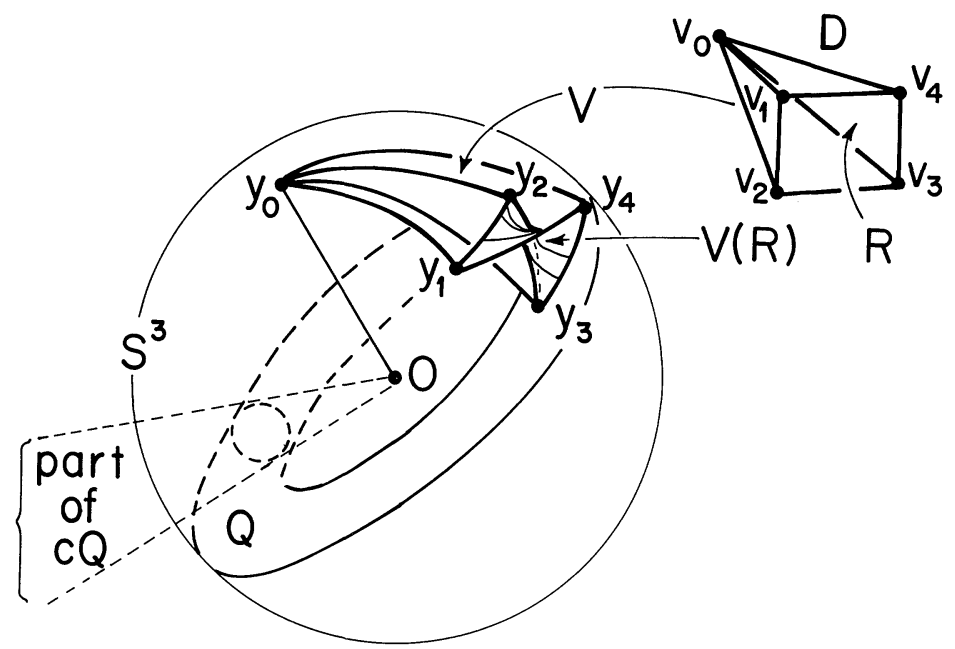

Fig. 4.2. The map $V$ of a pyramid $D$ into $S^{3}$

That is, if $X=\left(x_{1}, x_{2}, x_{3}, x_{4}\right)=\sum x_{i} y_{i}$ is regarded as a tangent vector at $z$, i.e. $X \in T_{z} \mathbf{R}^{4}=\mathbf{R}^{4}$, then it acts on $X$ by

$$
\left.\mathrm{d} q\right|_{z}(X)=t_{3} x_{1}-t_{4} x_{2}+t_{1} x_{3}-t_{2} x_{4} .
$$

4.31. (II.3) Our first constraints on $y$ are that it not lie in the boundary portion of $V(R)$. To ensure this we require that $y$ not lie in $c Q$, nor in any of the 3-planes through the origin determined by $\left(y_{0}, y_{1}, y_{2}\right)$, by $\left(y_{0}, y_{2}, y_{3}\right)$, by $\left(y_{0}, y_{3}, y_{4}\right)$ or by $\left(y_{0}, y_{1}, y_{4}\right)$.

The numerical criteria are that each of the following quantities be non-zero: $q\left(t_{1}(y), \ldots, t_{4}(y)\right), \operatorname{det}\left(y, y_{0}, y_{1}, y_{2}\right), \operatorname{det}\left(y, y_{0}, y_{2}, y_{3}\right), \operatorname{det}\left(y, y_{0}, y_{3}, y_{4}\right), \operatorname{det}\left(y, y_{0}, y_{1}\right.$, $\left.y_{4}\right)$.

4.32. Our other condition is that the geodesic circle through $y_{0}$ and $y$ not be tangent to $Q$. This is equivalent (see Fig. 4.3) to requiring that the line $L$ through $y$ parallel to $y_{0}$ not be tangent to $c Q$. We may parametrize $L$ as

Then

$$
L(t)=y-t y_{0} \text {. }
$$

$$
q(L(t))=t^{2} q\left(y_{0}\right)+\left.t \mathrm{~d} q\right|_{y_{0}}(y)+q(y) .
$$

Roots of this polynomial in $t$ give intersection points of $L$ with $c Q$; a tangency corresponds to a double root. The discriminant

$$
p(y)=\left[\left.\mathrm{d} q\right|_{y_{0}}(y)\right]^{2}-4 q\left(y_{0}\right) q(y)
$$

is homogeneous of order 2 in $y$, so the variety $\{p=0\}$ is a cone $c P$. Our final constraint on $y$ is that it not lie in $P=s(c P)$; it is sufficient to require $p(y) \neq 0$.

These six constraints exclude 2-dimensional sets in $S^{3}$, so the set of remaining $y$ 's is open and dense.

4.33. (II.4) We calculate $n(D)$ in three steps. First we count the number of points in which the geodesic semi-circle from $y_{0}$ through $y$ to $-y_{0}$ meets $Q$. We then see how 


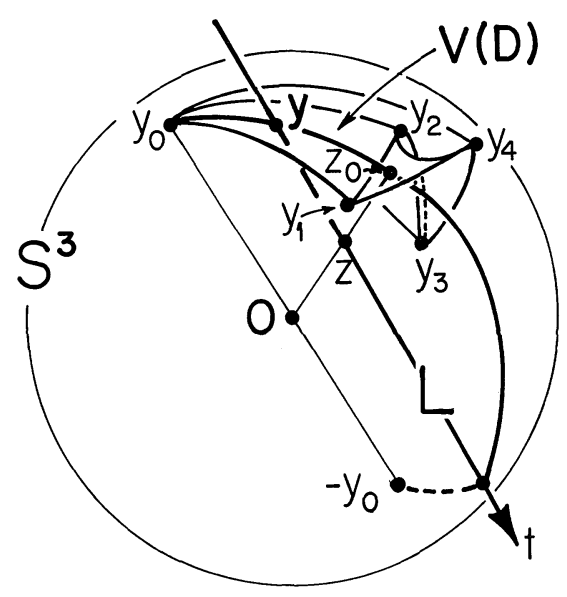

Fig. 4.3. A suitable choice of the point $y$ with respect to $V(D)$ when $D$ is a pyramid

many of these points actually lie in $V(R)$. Among such points we finally check which ones have $y$ between themselves and $y_{0}$.

Now $s(L)$ is the relative interior of the geodesic from $y_{0}$ through $y$ to $-y_{0}$. Since $y_{0}$ and $-y_{0}$ are not in $Q$, the number of points in which the geodesic meets $Q$ is equal to the number of points in which $L$ meets $c Q$. This is the number of solutions of $q(L(t))=0$. We have excluded the possibility of a repeated root, so there are either none or two, according as $p(y)<0$ or $>0$.

If $p(y)<0$ we are done, since then $n(D)=0$. So assume $p(y)>0$, and let $t^{\prime}$ and $t^{\prime \prime}$ be the roots of the equation $q(L(t))=0$. Then $s\left(L\left(t^{\prime}\right)\right)$ and $s\left(L\left(t^{\prime \prime}\right)\right)$ are the points where the semi-circle meets $Q$ (see Fig. 4.4). Now $s(L(t))$ lies in $V(R)$ if and only if all of its coordinates $t_{i}(s(L(t))) \geqq 0$. In fact, by our choice of $y$, none of them can be zero. Since $L(t)$ is a positive scalar multiple of $s(L(t))$, we may calculate the number of $t$ 's (this can be 0,1 , or 2 ) such that $q(L(t))=0$ and $t_{i}(L(t))>0$ for $i=1,2,3,4$. If this number is 0 , we are again finished, since $n(D)=0$. Otherwise, $y$ is between $y_{0}$ and $s(L(t))$ on the semi-circle if and only if $t$ is strictly positive.

4.34. To summarize, $n(D)$ is the number of real roots $t$ of the equation

$$
t^{2} q\left(y_{0}\right)-\left.t \mathrm{~d} q\right|_{y_{0}}(y)+q(y)=0
$$

such that

$$
t_{i}(L(t))>0, \quad i=1,2,3,4
$$

and

$$
t>0 \text {. }
$$

[Here $q$ is given by (4.28.1), $\mathrm{d} q$ by (4.30.1), $L(t)$ by (4.32.1) and $t_{i}(a)=\operatorname{det}_{i}(a$; $\left.y_{1}, y_{2}, y_{3}, y_{4}\right) / \operatorname{det}\left(y_{1}, y_{2}, y_{3}, y_{4}\right)$ are the coordinates of $a$ in the basis $y_{1}, y_{2}, y_{3}$, $y_{4}$.] 


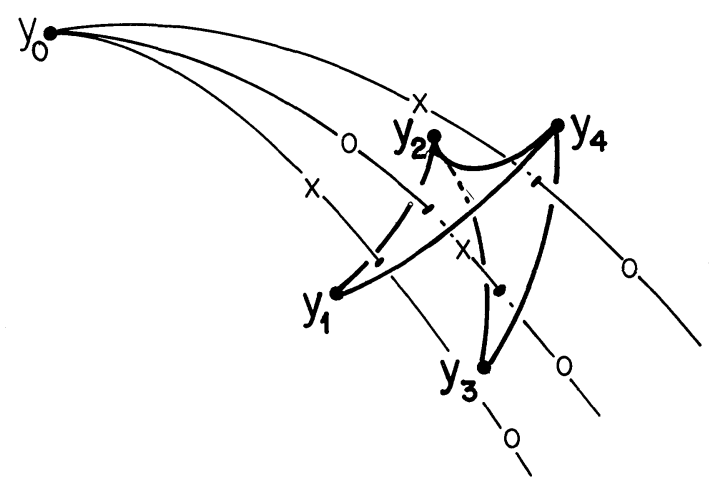

Fig. 4.4. Values of $n(D)$ for different choices of $y$. $D$ is a pyramid. When $y$ is at a point marked $x$, then $n(D)= \pm 1$; if $y$ is at a point marked $\circ$, then $n(D)=0$

4.35. (II.5) The configurations giving a nonzero $n(D)$ are shown in Fig. 4.4. The geodesic $s L(t)$ from $y_{0}$ to $y$ intersects the ruled surface $V(R)$ beyond $y$ in exactly one point $z_{0}$ [with $\left.\operatorname{dist}\left(y_{0}, z_{0}\right)<\pi\right]$. Our task is now to determine the appropriate sign $\varepsilon(D)$ : we assume that $v_{0} \ldots v_{4}$ is positively oriented (i.e. that the curve $v_{1} v_{2} v_{3} v_{4} v_{1}$, traversed as listed, gives the positive orientation on the boundary of the pyramid) and we calculate the sign of $\left.d V\right|_{x}$, where $x=V^{-1}(y)$.

Let $z$ be the corresponding intersection point of $L(t)$ with $c Q$, so the points $\mathbf{0}$, $y_{0}, y, z$ and $z_{0}$ are all in the same 2-plane in $\mathbf{R}^{4}$. Set $h(z)=\left.\operatorname{sign} d q\right|_{z}\left(z-y_{0}\right)$ $=-\left.\operatorname{sign} d q\right|_{z}\left(y_{0}\right)$. Since $\left.\operatorname{grad} q\right|_{z}$ is perpendicular to $c Q=\{q=0\}$, and since $\left.d q\right|_{z}\left(z-y_{0}\right)=\left\langle\left.\operatorname{grad} q\right|_{z}, z-y_{0}\right\rangle$, if this sign is positive it means that $L(t)$ is crossing at $z$ from $\{q<0\}$ to $\{q>0\}$ (in the direction of increasing $t$ ), and it gives us the same information about how the geodesic $s L(t)$ crosses $Q$ at $z_{0}$.

4.36. Suppose $\operatorname{sdet}\left(y_{1}, y_{2}, y_{3}, y_{4}\right)>0$. We may then simplify the argument by assuming that $y_{i}=e_{i}$, the $t^{t \text { th }}$ element of the standard quaternionic basis $\mathbf{1}, \mathbf{i}, \mathbf{j}, \mathbf{k}$ of $\mathbf{R}^{4}$. Then the function $q$ becomes $t_{1} t_{3}-t_{2} t_{4}$ in the standard $\mathbf{R}^{4}$ coordinates, and $\operatorname{grad} q$ $=\left(t_{3},-t_{4}, t_{1},-t_{2}\right)$. Projecting grad $q$ onto $S^{3}$ at 1 gives the vector $(0,1,0)=\mathbf{j} \in T S_{1}^{3}$. At $\mathbf{1}$ the tangent space to $V(R)$ is spanned by $\mathbf{i}$ and $\mathbf{k}$. If $1 \mathbf{i j k} \mathbf{1}$ is to be a positively oriented circuit on $V(R)$, then $\mathbf{i}, \mathbf{k}$ is a positive basis for $T V(R)_{\mathbf{1}}$. With respect to this basis $\mathbf{j}$ is a negative normal vector, since the basis $\mathbf{i}, \mathbf{j}, \mathbf{k}$ for $T S_{\mathbf{1}}^{3}$ gives the positive orientation of $S^{3}$; i.e. grad $q$ is a negative normal vector at $\mathbf{1}$ and therefore on all of $V(R)$. So in this case if $h(z)$ is positive then $s L(t)$ is crossing $V(\mathrm{R})$ in the negative direction at $z_{0}$, and $\varepsilon_{D}(x)=-1$.

4.37. In general,

$$
\varepsilon_{D}(x)=-h(z) \operatorname{sdet}\left(y_{1}, y_{2}, y_{3}, y_{4}\right),
$$

where $z$ is the intersection point of $L(t)=y-t y_{0}$ with $c Q$ and $h(z)$ is given in (4.35).

4.38. The last sentence of (4.35) gives a geometric interpretation of $h(z)$, from which it follows that if $n(D)=2$, then the two $\varepsilon$ 's cancel. This is in accordance with what we claimed in (5) of (4.18). 
4.39. Case III: $D$ is a Prism. There are two subcases. (a) $D$ is of type (1-simplex) $\times\left(2\right.$-simplex) These are the two prisms of $C^{2}$, where $V_{0 x}^{\Sigma}$ (which we shall continue to abbreviate as $V)$ is given by $V\left(s_{1}, s_{3}, s_{4}\right)=\mathfrak{g}_{012}\left(s_{1}\right) \mathfrak{h}_{234 \chi}\left(s_{3}, s_{4}\right)$. The two prisms of $C^{3}$ are of the form $D=(2$-simplex $) \times(1$-simplex $)$; this subcase (b) is similar and will be dealt with briefly after subcase (a).

We write $v_{1}, \ldots, v_{6}$ for the vertices of $D$ in the order listed in Table 4.1, and $y_{i}$ $=V\left(v_{i}\right)$ as usual.

One of the prisms of $C^{2}$ is $D=\Delta^{1} \times \Delta^{2}$, where $\Delta^{1}=\langle 02,012\rangle$ and $\Delta^{2}=\langle 0,23$, $234\rangle$. (The other has 24 instead of 23 .) $\Delta^{1}$ is parametrized by $0 \leqq s_{1} \leqq 1$, and $\Delta^{2}$ by $0 \leqq s_{3}, s_{4} \leqq 1, s_{3} \geqq s_{4}$. We will work on this prism, but state our results in terms of $y_{1}, \ldots, y_{6}$ so they will be applicable to both.

Let $V_{1}$ and $V_{2}$ denote the restrictions of $V$ to $\Delta^{1}$ and $\Delta^{2}$ respectively. $V_{1}$ maps $\Delta^{1}$ to the geodesic $s\left[u_{02}, u_{012}\right]$ and $V_{2}$ maps $\Delta^{2}$ to the convex spherical 2-simplex which we will write as $s\left[I, u_{23}, u_{234}\right]$, where as usual $u_{012}$ means $u_{01} u_{12}$, etc.

4.40. (IIIa.3) Our first task is to analyze the set of points at which $V$ is not a local diffeomorphism. (For this section and the next, refer to Fig. 4.5.) Let $\Sigma^{1}$ and $\Sigma^{2}$ represent the geodesic circle and 2-sphere determined by $V_{1}$ and $V_{2}$ respectively. Define $f: \Sigma^{1} \times \Sigma^{2}$ to be quaternionic multiplication, $f(\xi, n)=\xi \eta$. We want to know when, for $\xi \in \Sigma^{1}$ and $\eta \in \Sigma^{2}$,

$$
\left.\mathrm{d} f\right|_{(\xi, n)}: T_{(\xi, \eta)}\left(\Sigma^{1} \times \Sigma^{2}\right) \rightarrow T_{\xi \eta} S^{3}
$$

is not one-to-one. We may identify $T_{(\xi, \eta)}\left(\Sigma^{1} \times \Sigma^{2}\right)$ with $T_{(\xi \eta)}\left(\Sigma^{1} \cdot \eta\right) \oplus T_{(\xi \eta)}\left(\xi \cdot \Sigma^{2}\right)$. Now $f$ is a diffeomorphism on each of $\Sigma^{1} \cdot \eta$ and $\xi \cdot \Sigma^{2}$, so if $\left.\mathrm{d} f\right|_{(\xi, \eta)}$ is not one-toone, it must be the case that $T_{(\xi \eta)}\left(\Sigma^{1} \cdot \eta\right) \subset T_{(\xi \eta)}\left(\xi \cdot \Sigma^{2}\right)$. Since $\Sigma^{1} \cdot \eta$ is a geodesic and $\xi \cdot \Sigma^{2}$ is a great 2-sphere, this implies that $\Sigma^{1} \cdot \eta \subset \xi \cdot \Sigma^{2}$. If $\xi^{\prime}$ is any other point of $\Sigma^{1}$, left-multiplication by $\xi^{\prime} \xi^{-1}$ takes $\Sigma^{1}$ to itself; it follows that $\Sigma^{1} \cdot \eta \subset \xi^{\prime} \cdot \Sigma^{2}$; in particular $\xi \eta$ belongs to $u_{02} \cdot \Sigma^{2}$ and to $u_{012} \cdot \Sigma^{2}$. Conversely, if $\xi \eta$ is any point of $u_{02} \cdot \Sigma^{2} \cap u_{012} \cdot \Sigma^{2}$, then $\Sigma^{1} \cdot \eta \subset \xi \cdot \Sigma^{2}$. Thus $\left.\mathrm{d} f\right|_{(\xi, \eta)}$ fails to be one-to-one if and only if $\xi_{\eta}$ lies in $\Sigma^{\prime}=u_{02} \cdot \Sigma^{2} \cap u_{012} \cdot \Sigma^{2}$. The continuity condition implies that $\Sigma^{\prime}$ is a circle.

In conclusion, for $x \in D,\left.\mathrm{~d} V\right|_{x}$ is one-to-one provided $y=V(x)$ is not in $\Sigma^{\prime}$.

4.41. Our other constraint on $y$ is that it not lie in $V(\partial D)$. For $j=1,2,3$ let $\Sigma_{j}^{1}$ be the geodesic circle determined by $I, u_{23}, u_{234}$, leaving out the $j^{\text {th }}$ element. Then $V(\partial D)$ consists of portions of $u_{02} \cdot \Sigma^{2}, u_{012} \cdot \Sigma^{2}$, and $\Sigma^{1} \cdot \Sigma_{j}^{1}, j=1,2,3$. Since $\Sigma^{\prime} C u_{02} \cdot \Sigma^{2}$, the constraints on $y$ are all taken care of by requiring that $y$ not be in any of the five surfaces just mentioned.

Now for any $u \in S^{3}, y \notin u \cdot \Sigma^{2}$ provided $\operatorname{det}\left(y, u, u u_{23}, u u_{234}\right) \neq 0$. So we require:

$$
\operatorname{det}\left(y, y_{1}, y_{5}, y_{6}\right) \neq 0 \text { and } \operatorname{det}\left(y, y_{2}, y_{3}, y_{4}\right) \neq 0 \text {, }
$$

where $y_{i}=V\left(v_{i}\right)$ as before.

To detect whether or not $y$ is in $\Sigma^{1} \cdot \Sigma_{j}^{1}$ we could use functions of the type of $q: \mathbf{R}^{4} \rightarrow \mathbf{R}$ defined in Case II. But there is a simpler method, which is more easily explained in the course of showing how to calculate $n(D)$.

4.42. (IIIa.4) To calculate $n(D)$ we observe that $y \in s\left[u_{02}, u_{012}\right] \cdot s\left[I, u_{23}, u_{234}\right]$ if and only if the geodesic segment $s[z, y]$ intersects the great spherical triangle 

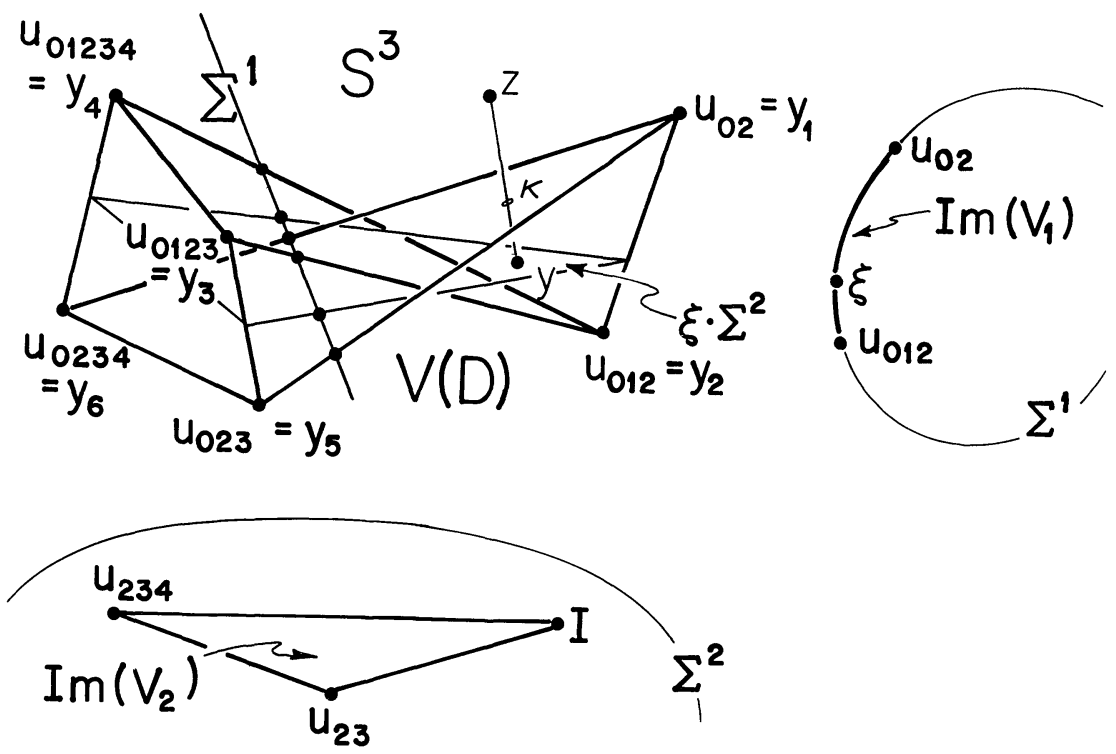

Fig. 4.5. A suitable choice of the point $y$ with respect to $V(D)$ when $D$ is a prism. (Geodesics in $\Sigma^{2}$ and $S^{3}$ are represented by straight lines)

$u_{02} \cdot s\left[I, u_{23}, u_{234}\right]$, where $z=u_{0210} y$. This can be detected in two steps. First we check whether or not $s[z, y]$ intersects $u_{02} \cdot \Sigma^{2}$. If they meet in a point $\kappa$, then by our choice of $y$ (so far) $\kappa$ is neither $z$ nor $y$, and $s[z, y]$ is not tangent at $\kappa$ to $u_{02} \cdot \Sigma^{2}$. It follows that $z$ and $y$ are on opposite sides of the 3-plane determined by $u_{02} \cdot \Sigma^{2}$.

Let $t_{1}, \ldots, t_{4}$ be coordinates on $\mathbf{R}^{4}$ with respect to $y, y_{1}, y_{5}$, and $y_{6}$. Then the 3 -plane of $u_{02} \cdot S^{2}$ is $\left\{t_{1}=0\right\}$, and $t_{1}(y)=1$. So $y \in s\left[u_{02}, u_{012}\right] \cdot S^{2}$ if and only if $t_{1}(z)<0$. Here

$$
t_{1}(z)=\operatorname{det}\left(z, y_{1}, y_{5}, y_{6}\right) / \operatorname{det}\left(y, y_{1}, y_{5}, y_{6}\right) \text {. }
$$

The numerator equals $\operatorname{det}\left(u_{0210} y, y_{1}, y_{5}, y_{6}\right)=\operatorname{det}\left(y, y_{2}, y_{3}, y_{4}\right)$ because quaternionic multiplication (by $u_{0210}$ in this case) is an orientation-preserving isometry. Hence our first necessary condition is

$y \in V(D)$ only if $\operatorname{det}\left(y, y_{2}, y_{3}, y_{4}\right) / \operatorname{det}\left(y, y_{1}, y_{5}, y_{6}\right)<0$.

Now to say that $y \in s\left[u_{02}, u_{012}\right] \cdot s\left[I, u_{23}, u_{234}\right]$ is to say that $\kappa \in u_{02} \cdot s\left[I, u_{23}\right.$, $\left.u_{234}\right]=s\left[u_{02}, u_{023}, u_{0234}\right]$. In other words, $t_{i}(\kappa) \geqq 0$ for $i=2,3,4$. In fact, if any $t_{i}(\kappa)=0$, then $\kappa \in \Sigma_{i}^{1}$, so $y \in \Sigma^{1} \cdot \Sigma_{i}^{1}$. So our extra constraints on $y$ in (4.41) are:

$$
t_{i}(\kappa) \neq 0 \text { for } i=2,3,4 .
$$

These constraints will be elucidated shortly.

The coordinates $t_{2}, t_{3}, t_{4}$ are in constant proportion on the 2-plane through $y$, $\kappa$, and the origin. These three coordinates are 0 at $y$; and since $\kappa$ is in the convex 


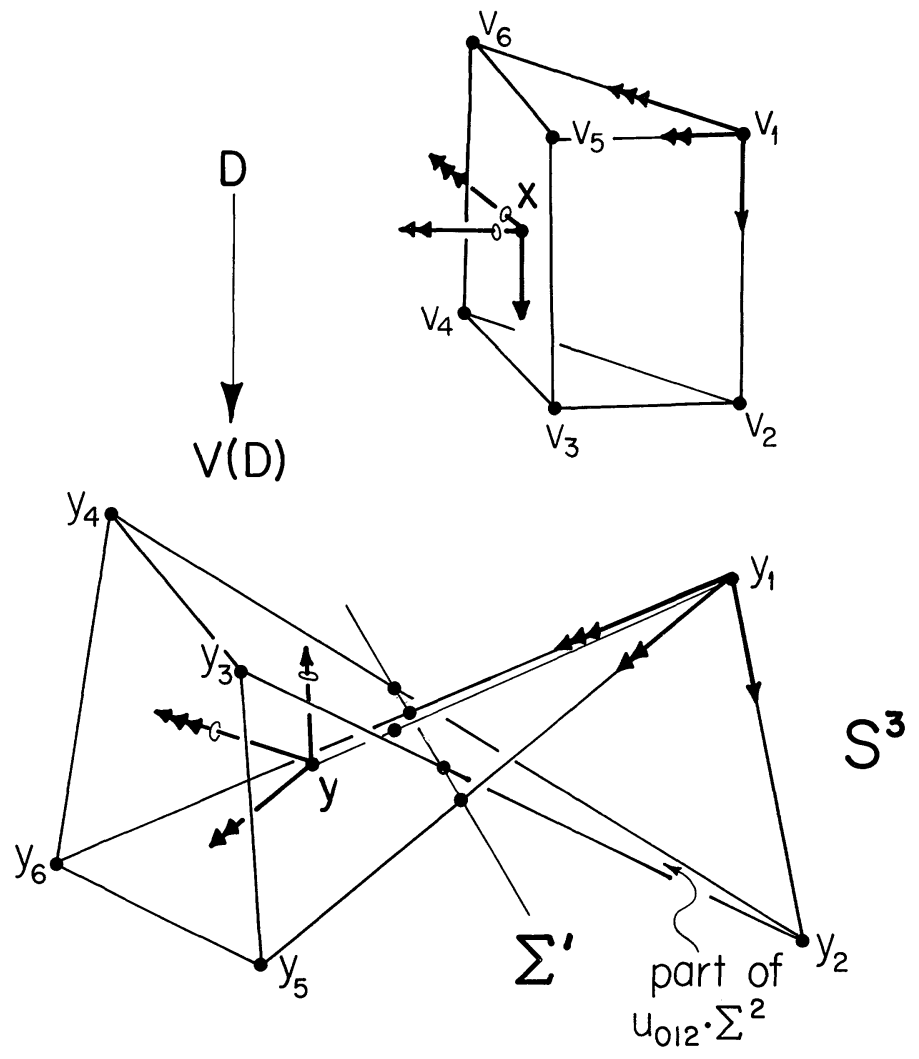

Fig. 4.6. Comparison of orientations at $y$ and $y_{1}$. (Geodesics in $S^{3}$ are represented by straight lines)

sector $\operatorname{cs}\left[u_{0210} y, y\right]$, they each have the same sign at $\kappa$ and at $u_{0210} y$. We may therefore require, in addition to (4.42.1),

$$
t_{i}\left(u_{0210} y\right)>0 \text { for } i=2,3,4 \text {. }
$$

Here, for example,

$$
t_{2}\left(u_{0210} y\right)=\operatorname{det}\left(y, u_{0210} y, y_{5}, y_{6}\right) / \operatorname{det}\left(y, y_{1}, y_{5}, y_{6}\right) .
$$

If these conditions are met, then $y$ can be written as $\xi \eta$, with $\xi \in s\left[u_{02}, u_{012}\right]$ and $\eta \in s\left[I, u_{23}, u_{234}\right]$. Here $\xi$ and $\eta$ are uniquely determined by $y$; and since $V_{1}$ and $V_{2}$ are diffeomorphisms, it follows that the coordinates $s_{1}$ and $\left(s_{3}, s_{4}\right)$ of a point $x$ in $D$ such that $V(x)=y$ are also uniquely determined. Of course if any of the conditions is violated, then $n(D)=0$.

4.44. (IIIa.5) It is clear from Fig. 4.6 that if $\Sigma^{\prime} \cap V(D) \neq \emptyset$, then the sign of d $\left.V\right|_{x}$ depends on the position of $y$ with respect to $\Sigma^{\prime}$. In fact sign $\left.\mathrm{d} V\right|_{x}=\left.\operatorname{signd} V\right|_{v_{1}}$ if the geodesic segment $s\left[y_{1}, y\right]$ does not intersect $u_{012} \cdot \Sigma^{2}$, i.e. if $y$ and $y_{1}$ are on the 
same side of the 3-plane through $\mathbf{0}$ determined by $y_{2}, y_{3}$, and $y_{4}$; equivalently, if $\operatorname{det}\left(y, y_{2}, y_{3}, y_{4}\right) \operatorname{det}\left(y_{1}, y_{2}, y_{3}, y_{4}\right)>0$. To calculate the sign of $\left.\mathrm{d} V\right|_{v_{1}}$, observe that since $\partial / \partial s_{1}, \partial / \partial s_{3}, \partial / \partial s_{4}$ form a positively oriented basis at 02 , then so do $v_{1} v_{2}, v_{1} v_{5}$, $v_{1} v_{6}$; so the sign of $\left.\mathrm{d} V\right|_{v_{1}}$ is the sign in $S^{3}$ of the frame $y_{1} y_{2}, y_{1} y_{5}, y_{1} y_{6}$, i.e. $\operatorname{sdet}\left(y_{1}\right.$, $\left.y_{2}, y_{5}, y_{6}\right)$. Finally

$$
\varepsilon_{D}(x)=\operatorname{sdet}\left(y, y_{2}, y_{3}, y_{4}\right) \operatorname{sdet}\left(y_{1}, y_{2}, y_{3}, y_{4}\right) \operatorname{sdet}\left(y_{1}, y_{2}, y_{5}, y_{6}\right) .
$$

4.45. Case IIIb. Here $D=\Delta^{2} \times \Delta^{1}$, where in one prism $\Delta^{2}=\langle 03,013,0123\rangle$ and $\Delta^{1}$ $=\langle 0,34\rangle$ (in the other, $\Delta^{2}=\langle 03,023,0123\rangle$ ). Again, we will work with the first prism but give results in a form applicable to both. The vertices of $D$, in the order in which they are listed in Table 4.1, are $v_{1}=03, v_{2}=013, v_{3}=0123, v_{4}=01234, v_{5}$ $=034, v_{6}=0134 . D$ is part of a cube parametrized by $s_{1}, s_{2}$, and $s_{4} ; \Delta^{1}$ is parametrized by $s_{4}$ and $\Delta^{2}$ by $\left(s_{1}, s_{2}\right), s_{1} \geqq s_{2}$. As before, $V\left(s_{1}, s_{2}, s_{4}\right)=V_{2}\left(s_{1}, s_{2}\right)$ - $V_{1}\left(s_{4}\right)$. The argument is now exactly as in case (a), except that the order of multiplication in $S^{3}$ is systematically reversed.

4.46. (IIIb.3) Let $\Sigma^{2}=s\left[u_{03}, u_{013}, u_{0123}\right]$ and $\Sigma^{1}=s\left[I, u_{34}\right]$. We define f : $\Sigma^{2} \times \Sigma^{1}$ $\rightarrow S^{3}$ by $f(\eta, \xi)=\eta \xi$. We find that $\left.d f\right|_{(\eta, \xi)}: T_{(\eta, \xi)}\left(\Sigma^{2} \times \Sigma^{1}\right) \rightarrow T_{\eta \xi} S^{3}$ is one-to-one unless $\eta \xi$ is on the circle $\Sigma^{\prime}=\Sigma^{2} \cap \Sigma^{2} \cdot u_{34}$, and $\left.d V\right|_{x}: T_{x} D \rightarrow T_{V(x)} S^{3}$ is one-to-one provided $y=V(x)$ is not in $\Sigma^{\prime}$. The constraint that $\left.d V\right|_{x}$ be one-to-one at $x=V^{-1} y$ is thus included in the requirement that $y$ not be in the portion of $V(\partial D)$ given by $s\left[y_{1}, y_{2}, y_{3}\right]$; it is sufficient that $\operatorname{det}\left(y, y_{1}, y_{2}, y_{3}\right) \neq 0$. Similarly, $y$ will not lie in the opposite triangular face if $\operatorname{det}\left(y, y_{4}, y_{5}, y_{6}\right) \neq 0$.

The other constraints on $y$ are that it not lie in the three lateral portions of $V(\partial D)$. These can be guaranteed by

$$
\begin{aligned}
& \operatorname{det}\left(y, y u_{43}, u_{03}, u_{013}\right) \neq 0, \\
& \operatorname{det}\left(y, y u_{43}, u_{013}, u_{0123}\right) \neq 0, \\
& \operatorname{det}\left(y, y u_{43}, u_{0123}, u_{03}\right) \neq 0 .
\end{aligned}
$$

4.47. (IIIb.4) The condition that $y \in \Sigma^{2} \cdot s\left[I, u_{34}\right]$ is $t_{1}(z)<0$, where $t_{1}, \ldots, t_{4}$ are coordinates on $\mathbf{R}^{4}$ with respect to $y, y_{1}, y_{2}$, and $y_{3}$, and where $z=y u_{43}$. Now

$$
t_{1}(z)=\operatorname{det}\left(z, y_{1}, y_{2}, y_{3}\right) / \operatorname{det}\left(y, y_{1}, y_{2}, y_{3}\right) \text {, }
$$

so our condition is

$$
\operatorname{det}\left(y, y_{5}, y_{6}, y_{4}\right) / \operatorname{det}\left(y, y_{1}, y_{2}, y_{3}\right)<0 \text {. }
$$

To ensure that $y \in V(D)$ we must further require $t_{i}(z)>0$, for $i=2,3,4$. Here, for example,

$$
t_{2}(z)=\operatorname{det}\left(y, y u_{43}, y_{2}, y_{3}\right) / \operatorname{det}\left(y, y_{1}, y_{2}, y_{3}\right) .
$$

If these conditions are satisfied, then $n(D)=1$; otherwise, $n(D)=0$. 
4.48. (IIIb.5) Arguing as in (4.44) we obtain

$$
\varepsilon_{D}(x)=\operatorname{sdet}\left(y, y_{4}, y_{5}, y_{6}\right) \operatorname{sdet}\left(y_{1}, y_{4}, y_{5}, y_{6}\right) \operatorname{sdet}\left(y_{1}, y_{2}, y_{3}, y_{5}\right)
$$

Acknowledgements. The second author is grateful to the Science and Engineering Research Council (U.K.) for support under Award GR/C/57891, and to the University College of London University for hospitality during the summer of 1984.

\section{References}

1. Bott, R., Tu, L.: Differential forms in algebraic topology. Berlin, Heidelberg, New York: Springer 1982

2. Christ, N.H., Friedberg, R., Lee, T.D.: Random lattice field theory; general formulation. Nucl. Phys. B 202, 89-125 (1982)

3. Christ, N.H., Friedberg, R., Lee, T.D.: Gauge theory on a random lattice. Nubl. Phys. B 210 [FS6], 310-336 (1982)

4. Christ, N.H., Friedberg, R., Lee, T.D.: Weights of links and plaquettes in a random lattice. Nucl. Phys. B 210 [FS6], 337-346 (1982)

5. Cotta-Ramusino, P., Dell'Antonio, G.: Selfduality and topological-like properties of lattice gauge field theories. A proposal. Commun. Math. Phys. 70, 75-95 (1979)

6. DiVecchia, P., Fabricius, K., Rossi, G.C., Veneziano, G.: Preliminary evidence for $U_{A}(1)$ breaking in QCD from lattice calculations. Nucl. Phys. B 192 392-408 (1981)

7. Drouffe, J., Moriarity, K.: Gauge theories on a simplicial lattice. Nucl. Phys. B 220, 253-269 (1983)

8. Eguchi, T., Gilkey, P., Hanson, A.: Gravitation, gauge theories, and differential geometry. Phys. Rep. 66, 213-393 (1980)

9. Fox, I., Gilchrist, J., Laursen, M., Schierholz, G.: The topological charge in SU(2) lattice gauge theory. Phys. Rev. Lett. 54, 749-752 (1985)

10. Flume, R., Wyler, D.: Topological charge in the lattice Schwinger model. Phys. Lett. 108 B, 317-322 (1981)

11. Greenberg, M.: Lectures on algebraic topology. New York: Benjamin 1967

12. Hirsch, M.: Differential topology. Berlin, Heidelberg, New York: Springer 1976

13. Ishikawa, K., Schierholz, G., Schneider, H., Teper, M.: On the topological structure of the vacuum in SU(2) and SU(3) lattice gauge theories. Phys. Lett. 128 B, 309-315 (1983)

14. Iwasaki, Y., Yoshie, T.: Instantons and topological charge in lattice gauge theory. Phys. Lett. $131 \mathrm{~B}, 159-164$ (1983)

15. Lasher, G., Phillips, A., Stone, D.: A reliable combinatorial algorithm for the topological charge of SU(2) lattice gauge fields, to appear in Proceedings, Conference on Quark Confinement and Liberation, Lawrence Berkeley Laboratory, May 1985

16. Levitt, N., Rourke, C.: The existence of combinatorial formulae for characteristic classes. Trans. Am. Math. Soc. 239, 391-397 (1978)

17. Lüscher, M.: A semi-classical formula for the topological susceptibility in a finite space-time volume. Nucl. Phys. B 205 [FS5], 483-503 (1982)

18. Lüscher, M.: Topology of lattice gauge fields. Commun. Math. Phys. 85 39-48 (1982)

19. Milnor, J.: Characteristic classes. Annals of mathematics studies, Vol. 76. Princeton, NJ: Princeton University Press 1974

20. Nijenhuis, A.: On the holonomy groups of linear connections. I. A, B General properties of affine connections. Indagationes Math. 15, 233-249 (1953); II. Properties of general linear connections. Indagationes Math. 16, 17-25 (1954)

21. Panagiotakopoulos, C.: Topology of 2D lattice gauge fields. Nucl. Phys. B 251 [FS13], 61-76 (1985) 
22. Panagiotakopoulos, C.: Topology of 4D lattice gauge fields. Rockefeller University preprint RU 85/B/109

23. Phillips, A.: Characteristic numbers of $U_{1}$-valued lattice fields. Ann. Phys. 161, 399-422 (1985)

24. Polonyi, J.: On the topological charge of lattice gauge theories. Phys. Rev. D 29, 716-721 (1984)

25. Polyakov, A.M.: Compact gauge fields and the infrared catastrophe. Phys. Lett. 59 B, 82-84 (1975)

26. Schlesinger, L.: Parallelverschiebung und Krümmungstensor. Math. Ann. 99, 413-434 (1928)

27. Steenrod, N.: Topology of fibre bundles. Princeton, NJ: Princeton University Press, 1951

28. Wallace, A.: Algebraic topology; homology and cohomology. New York: Benjamin 1970

29. Wilson, K.: Confinement of quarks. Phys. Rev. D 10, 2445-2459 (1974)

30. Woit, P.: Topological charge in lattice gauge theory. Phys. Rev. Lett. 51, 638-641 (1983)

31. Woit, P.: Topology and lattice gauge fields, Stony Brook ITP preprint

Communicated by K. Osterwalder

Received June 26, 1985; in revised form October 7, 1985 